\title{
Statistical inference of the stress-strength reliability and mean remaining strength of series system with cold standby redundancy at system and component levels
}

\author{
Gülce Cüran ${ }^{1}$ (ID), Fatih Kızılaslan*2 (D) \\ ${ }^{1}$ Department of Mathematics, Yeditepe University, Istanbul, Turkey \\ ${ }^{2}$ Department of Statistics, Marmara University, Istanbul, Turkey
}

\begin{abstract}
In this study, we consider the stress-strength reliability and mean remaining strength of a series system with cold standby redundancy at the component and system levels. Classical and Bayesian approaches are studied in order to obtain the estimates when the underlying stress, strength and standby components follow the exponential distribution with different parameters. Bayes estimates are approximated by using Lindley's approximation and Markov Chain Monte Carlo methods. Asymptotic confidence intervals and highest probability density credible intervals are constructed. We perform Monte Carlo simulations to compare the performance of proposed estimates. A real data set is analyzed for the purpose of illustration.
\end{abstract}

Mathematics Subject Classification (2020). 62N05, 62F15

Keywords. Stress-strength reliability, mean remaining strength, cold standby, series system

\section{Introduction}

In its simplest form, the stress-strength model describes the reliability of a component or system in terms of random variables. In this case, the reliability is defined as $P(X<Y)$ where $X$ is the random stress experienced by the system, and $Y$ is the random strength of the system available to overcome the stress. The system fails if the stress exceeds the strength. This main idea was introduced by [8] and developed by [9]. Estimation problem for the reliability of a coherent system such as simple, series, parallel, and multicomponent systems has attracted a great deal attention in reliability literature. Some recent research contributions to the topic can be found in $[1,2,7,11,23-25,36]$.

In the stress-strength model, it is possible to learn how long the component or system can be safe under the stress on the average. The mean remaining strength (MRS) of the component or system is defined as the expected remaining strength under the stress $X$, i.e. $\Phi=E(Y-X \mid Y>X)$. The MRS of a system in stress-strength model is introduced by [19]. There are no works on a study for the estimation of the MRS except [20] and [26].

*Corresponding Author.

Email addresses: gulceulupinar@gmail.com (G. Cüran), fatih.kizilaslan@marmara.edu.tr (F. Kızılaslan) Received: 02.10.2020; Accepted: 24.07.2021 
A coherent system is an important concept in reliability theory and survival analysis. It contains well known systems such as series, parallel and $k$-out-of- $n$ systems. Interested readers are referred to the excellent monograph by [6] for more details. An efficient method for optimizing the lifetime of a coherent system is to add redundancy components (or spares) to the system. Three types of redundancies are commonly used in the reliability literature, i.e., active redundancy (hot standby), standby redundancy (cold standby), and warm standby. In the hot standby, available redundancy components are put in parallel with the original components and function simultaneously with them. In the cold standby, redundancy components are put in standby and start functioning when the original components fail. The warm standby is a redundancy type between the hot standby and the cold standby. It is called general standby because it contains both the hot standby and the cold standby. The warm standby case was studied by $[12,16]$.

The structure function $\phi$ of $n$-component system maps the state vector of the components of a given system to the state of system, i.e. $\phi:\{0,1\}^{n} \rightarrow\{0,1\}$ where 1 and 0 mean a component works and fails, respectively. A system is called coherent if its structure function $\phi$ is nondecreasing in each argument, and each component is relevant to the performance of the system. The lifetime $T$ of a coherent system based on components with lifetimes $X_{1}, \ldots, X_{n}$ can be written as $T=\phi\left(X_{1}, \ldots, X_{n}\right)$. For example, the structure function of series and parallel systems are $\phi\left(X_{1}, \ldots, X_{n}\right)=\min \left(X_{1}, \ldots, X_{n}\right)$ and $\phi\left(X_{1}, \ldots, X_{n}\right)=\max \left(X_{1}, \ldots, X_{n}\right)$, respectively. For more details on the theory of coherent systems, we refer the reader to the classic book Barlow and Proschan [6].

The performance of a coherent system consisting of $n$ independent components can be improved by adding $n$ standby redundancy components to each of the original components or creating a duplicate system consisting of standby components similar to the original coherent system. For instance, we have a series system with $n$ components with $n$ standby redundancy components. In this case, these standby components can be used either at component level or system level (see Figure 1 in [41]).

Standby redundancy can be applied at system level or component level. The lifetime of the system after standby redundancy at system level is $T_{S}=\phi\left(X_{1}, \ldots, X_{n}\right)+$ $\phi\left(Y_{1}, \ldots, Y_{n}\right)$, where $X_{i}$ and $Y_{i}$ are the lifetime of component $i$ and standby component $i$, respectively. The lifetime of the system after standby redundancy at component level is $T_{C}=\phi\left(X_{1}+Y_{1}, \ldots, X_{n}+Y_{n}\right)$. For the series system, the lifetimes become $T_{S}=\min \left(X_{1}, \ldots, X_{n}\right)+\min \left(Y_{1}, \ldots, Y_{n}\right)$ and $T_{C}=\min \left(X_{1}+Y_{1}, \ldots, X_{n}+Y_{n}\right)$.

It is clear that adding a standby component(s) to the system increases the system reliability. That is why system engineers want to answer which type of standby redundancy gives a longer lifetime for a $n$-component system. It is proved that standby redundancy is more effective at the component (system) level for a series (parallel) system by [37]. Different studies have been considered by many researchers in the reliability literature. For example, stochastic comparisons of the series and parallel systems which standby redundancy at component and system levels were studied by $[10,22,27]$. Some properties of multi-state series and cold standby systems consisting of two components was investigated by [15]. The effectiveness of adding cold standby redundancy to a coherent system at system and component level was investigated by [17]. How or where to allocate redundancy components in a coherent system is another interesting problem. Interested readers may refer to $[13,40,41]$.

To the best of our knowledge, the reliability estimation problem of the stress-strength model for cold standby in series or parallel systems has not been paying much attention except the following two papers. Estimation of the stress-strength reliability for a parallel system consists of active, warm and cold standby components was studied by [38]. When the standby redundancy system consists of a certain number of same subsystems with series structure, the reliability estimation of this system was considered by [30] for the generalized half-logistic distribution based on progressive Type-II censoring sample. 
In this study, we consider the stress-strength reliability and MRS of the series system when the standby components are applied at system level and component level. The main contribution of this study is to investigate the performance of the stress-strength reliability and MRS estimators for the series system for both cold standby cases. It is assumed that all the system components and standby components are independent but not identically distributed random variables belonging to the exponential distribution. Moreover, the stress-strength reliability of the interested series system has been compared with the series system without standby components.

The rest of this study is organized as follows. In Section 2, we present our model and some distributional properties. In Section 3, we obtain maximum likelihood estimate (MLE) and Bayesian estimate of the stress-strength reliability and MRS for the series system with component level redundancy. Lindley's approximation and Markov Chain Monte Carlo (MCMC) methods using hybrid Metropolis-Hastings and Gibbs sampling algorithm are implemented under Bayesian estimation. In Section 4, we derive ML and MCMC estimates of the stress-strength reliability and MRS for the series system with system level redundancy. The asymptotic confidence intervals and the highest probability density (HPD) credible intervals of the stress-strength reliability and MRS are also constructed in Sections 3 and 4. In Section 5, we carry out a simulation study to compare the performance of the aforementioned estimates for the stress-strength reliability and MRS. In Section 6, we present analyses of a real data set for illustrative purposes. Finally, we conclude the paper with some remarks in Section 7.

\section{Model description}

Consider a series system with $n$-components having independent and identically lifetime distribution. In this system, it is assumed that $X_{1}, \ldots, X_{n}$ are the lifetimes of strength components and follow the exponential distribution with parameter $\alpha$. Suppose that $Y_{1}, \ldots, Y_{n}$ are the lifetimes of independent standby strength components in the series system and follow the exponential distribution with parameter $\beta$. These standby redundancy components can be added to the series system at component level or system level. Suppose that $T$ is the common stress variable that is experienced by the series system and follows the exponential distribution with parameter $\theta$.

For the series system at component level, the standby redundancy components are added for each particular component of the series system. In this case, the total lifetime of each strength component is denoted by $Z_{i}=X_{i}+Y_{i}, i=1, \ldots, n$. Then, the cumulative distribution function $(\mathrm{CDF})$ and probability density function $(\mathrm{PDF})$ of $Z_{i}, i=1, \ldots, n$ are given by

$$
\begin{aligned}
F_{Z_{i}}(z) & =\int_{0}^{z} F_{x}(z-y) f_{y}(y) d y \\
& =\left\{\begin{array}{ll}
1+\frac{\alpha e^{-\beta z}-\beta e^{-\alpha z}}{\beta-\alpha}, & \alpha \neq \beta \\
1-e^{-\alpha z}(1+\alpha z), & \alpha=\beta
\end{array},\right.
\end{aligned}
$$

and

$$
f_{Z_{i}}(z)=\left\{\begin{array}{ll}
\frac{\alpha \beta}{\beta-\alpha}\left(e^{-\alpha z}-e^{-\beta z}\right), & \alpha \neq \beta \\
\alpha^{2} z e^{-\alpha z}, & \alpha=\beta
\end{array} .\right.
$$

It is clear that $Z_{i}, i=1, \ldots, n$ follow the Gamma distribution with parameters $\alpha$ and 2 when the active strength and standby redundancy components are identical, i.e. $\alpha=\beta$. Since $T$ is the common stress variable in the series system consisting of $Z_{i}, i=1, \ldots, n$, 
the stress-strength reliability of this system is obtained as

$$
\begin{aligned}
R_{\text {Comp }} & =P\left(Z_{(1)}>T\right)=\prod_{i=1}^{n} \int_{0}^{\infty}\left(1-F_{Z_{i}}(t)\right) f_{T}(t) d t \\
& = \begin{cases}\frac{1}{(\beta-\alpha)^{n}} \sum_{k=0}^{n}\left(\begin{array}{l}
n \\
k
\end{array}\right)(-1)^{k} \frac{\alpha^{k} \beta^{n-k} \theta}{[\alpha(n-k)+\beta k+\theta]}, & \alpha \neq \beta \\
\sum_{k=0}^{n}\left(\begin{array}{l}
n \\
k
\end{array}\right) \Gamma(k+1) \frac{\alpha^{k} \theta}{(\alpha n+\theta)^{k+1}}, & \alpha=\beta\end{cases}
\end{aligned}
$$

where $Z_{(1)}=\min \left(Z_{1}, \ldots, Z_{n}\right)$ and $\Gamma($.$) is the Gamma function.$

For the series system at system level, the standby redundancy components constitute a duplicate series system for the original series system. In this case, the total lifetime of these series systems becomes $Z_{(1)}=X_{(1)}+Y_{(1)}$ where $X_{(1)}$ and $Y_{(1)}$ follow the exponential distributions with parameters $n \alpha$ and $n \beta$, respectively. Then, the pdf and cdf of $Z_{(1)}$ are given by

$$
\begin{aligned}
f_{Z_{(1)}}(z) & =\int_{0}^{z} f_{X_{(1)}}(z-y) f_{Y_{(1)}}(y) d y \\
& =\frac{n \alpha \beta}{\beta-\alpha}\left(e^{-\alpha n z}-e^{-\beta n z}\right),
\end{aligned}
$$

and

$$
\begin{aligned}
F_{Z_{(1)}}(z) & =\int_{0}^{z} \frac{n \alpha \beta}{\beta-\alpha}\left(e^{-\beta t n}-e^{-\alpha t n}\right) d t \\
& =1+\frac{\alpha e^{-\beta n z}-\beta e^{-\alpha n z}}{\beta-\alpha},
\end{aligned}
$$

for $\alpha \neq \beta$. Also, $Z_{i}, i=1, \ldots, n$ follow the Gamma distribution with parameters $n \alpha$ and 2 for $\alpha=\beta$. Under the common stress variable $T$, the stress-strength reliability of this system is obtained as

$$
R_{\text {System }}=P\left(Z_{(1)}>T\right)=\frac{\theta(n(\beta+\alpha)+\theta)}{(\alpha n+\theta)(\beta n+\theta)},
$$

for any $\alpha$ and $\beta$. In the following sections, the equal strength parameters case $(\alpha=\beta)$ has not been considered. In that case, the reliability problem is similar to the simple stress strength reliability of the Gamma components. For more details about this case, see $[4,33]$.

Moreover, we consider $n$-components series system without standby components in the stress-strength model. Let $X_{1}, \ldots, X_{n}$ be the lifetimes of strength components which follow the exponential distribution with parameter $\alpha$, and $T$ is the common stress variable follow the exponential distribution with parameter $\theta$. In this case, $n$ strength components constitute a series system, and the lifetime of series system is $X_{(1)}$ follow the exponential distribution with parameter $n \alpha$. Then, the stress-strength reliability of this series system is

$$
R=P\left(X_{(1)}>T\right)=\frac{\theta}{\alpha n+\theta} .
$$

It is known that adding standby components increases system reliability. We want to show how these standby components affect the stress-strength reliability of the system in our case with graphs. In Figure 1, the stress-strength reliability values of the aforementioned three different series systems are plotted by using Equations (2.3), (2.6) and (2.7) based on different parameter sets. It is seen that standby redundancy increases system reliability. Therefore, the standby systems can be preferable under suitable circumstances. 

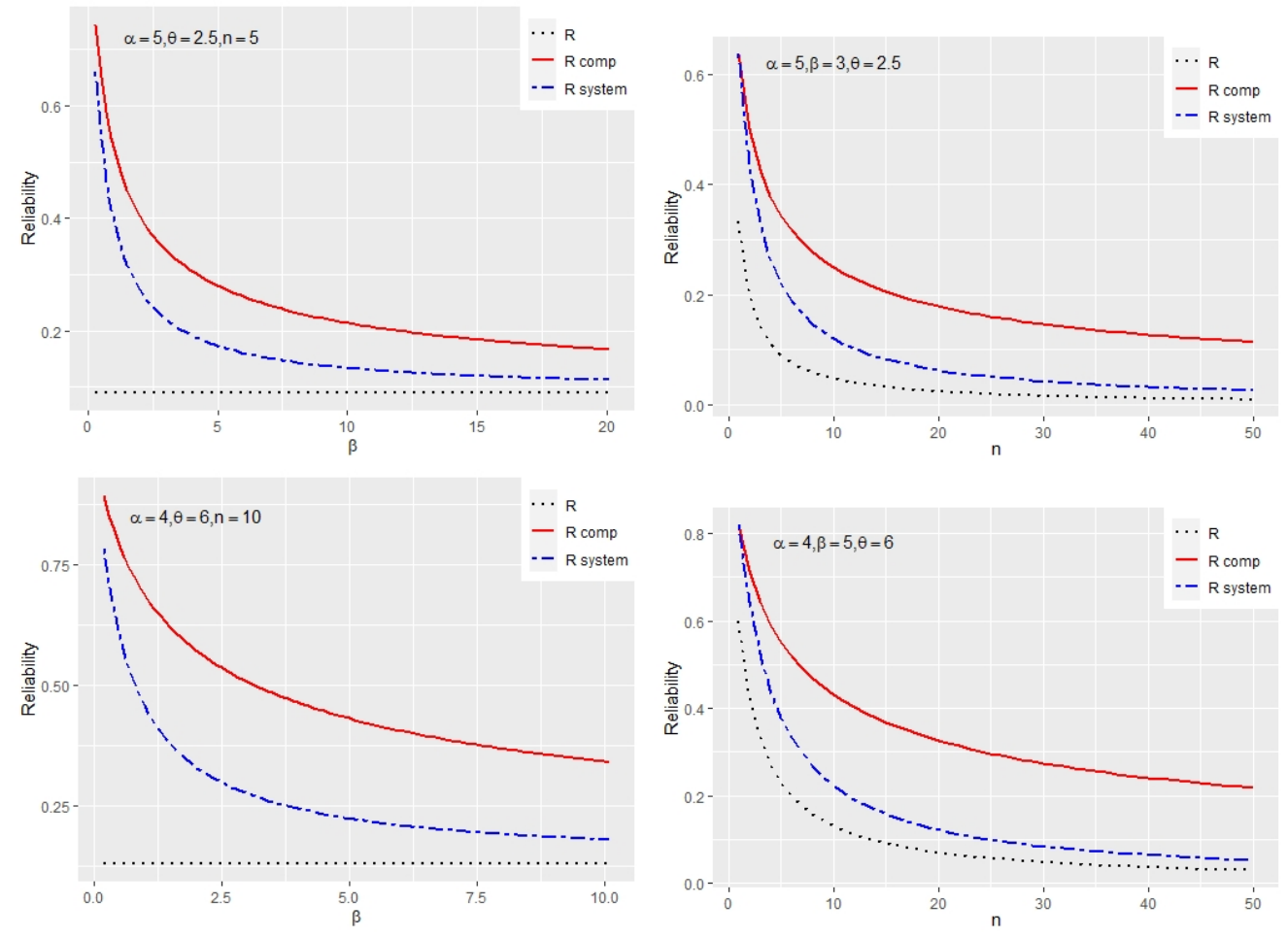

Figure 1. Plots for the reliabilities of $R, R_{C o m p}$ and $R_{\text {System }}$.

\section{Estimation of $R_{C o m p}$ and $\Phi_{C o m p}$}

In this section, point and interval estimations of the stress-strength reliability and MRS of the series system with cold standby at component level are investigated.

\subsection{MLE of $R_{C o m p}$}

Suppose that $m$ systems are put on a test each with $n$ original components and $n$ cold standby components in the series system. Then, the strength data is represented as $Z_{i 1}, \ldots, Z_{i n}, i=1, \ldots, m$ and stress is $T_{i}, i=1, \ldots, m$. The likelihood function of $\alpha, \beta$ and $\theta$ for the observed sample is

$$
\begin{aligned}
L(\alpha, \beta, \theta ; \mathbf{z}, \mathbf{t}) & =\prod_{i=1}^{m}\left(\prod_{j=1}^{n} f_{Z}\left(z_{i j}\right)\right) f_{T}\left(t_{i}\right) \\
& =\left(\frac{\alpha \beta}{\beta-\alpha}\right)^{n m} \exp \left(\sum_{i=1}^{m} \sum_{j=1}^{n} \ln \left(e^{-\alpha z_{i j}}-e^{-\beta z_{i j}}\right)\right) \theta^{m} e^{-\theta \sum_{i=1}^{m} t_{i}} .
\end{aligned}
$$

The corresponding log-likelihood function is

$$
\ell(\alpha, \beta, \theta ; \mathbf{z}, \mathbf{t})=n m(\ln (\alpha \beta)-\ln (\beta-\alpha))+m \ln \theta+\sum_{i=1}^{m} \sum_{j=1}^{n} \ln \left(e^{-\alpha z_{i j}}-e^{-\beta z_{i j}}\right)-\theta \sum_{i=1}^{m} t_{i} .
$$

By partially differentiating Equation (3.1) with respect to $\alpha$ and $\beta$, we obtain the following likelihood equations as

$$
\begin{aligned}
& \frac{\partial \ell}{\partial \alpha}=n m\left(\frac{1}{\alpha}+\frac{1}{\beta-\alpha}\right)-\sum_{i=1}^{m} \sum_{j=1}^{n} \frac{z_{i j} e^{-\alpha z_{i j}}}{e^{-\alpha z_{i j}}-e^{-\beta z_{i j}}}=0, \\
& \frac{\partial \ell}{\partial \beta}=n m\left(\frac{1}{\beta}-\frac{1}{\beta-\alpha}\right)+\sum_{i=1}^{m} \sum_{j=1}^{n} \frac{z_{i j} e^{-\beta z_{i j}}}{e^{-\alpha z_{i j}}-e^{-\beta z_{i j}}}=0 .
\end{aligned}
$$


The MLEs of the parameters $\alpha$ and $\beta$, i.e. $\widehat{\alpha}$ and $\widehat{\beta}$, can be obtained by solving the nonlinear equations in (3.2) and (3.3) simultaneously. This non-linear equation system can be solved by using numerical methods such as Newton-Raphson and Broyden's methods. The MLE of $\theta$ is derived as $\widehat{\theta}=m / \sum_{i=1}^{m} t_{i}$. After obtaining $\widehat{\alpha}, \widehat{\beta}$ and $\widehat{\theta}$, the MLE of $R_{C o m p}$, i.e. $\widehat{R}_{C o m p}^{M L E}$, is computed from Equation (2.3) by using the invariance property of MLE.

\subsection{Asymptotic confidence interval of $R_{C o m p}$}

The observed information matrix of $\tau=(\alpha, \beta, \theta)$ is defined as

$$
J(\tau)=-\left(\begin{array}{ccc}
\frac{\partial^{2} \ell}{\partial \alpha^{2}} & \frac{\partial^{2} \ell}{\partial \alpha \partial \beta} & \frac{\partial^{2} \ell}{\partial \alpha \partial \theta} \\
\frac{\partial^{2} \ell}{\partial \beta \partial \alpha} & \frac{\partial^{2} \ell}{\partial \beta^{2}} & \frac{\partial^{2} \ell}{\partial \beta \partial \theta} \\
\frac{\partial^{2} \ell}{\partial \theta \partial \alpha} & \frac{\partial^{2} \ell}{\partial \theta \partial \beta} & \frac{\partial^{2} \ell}{\partial \theta^{2}}
\end{array}\right)=\left(\begin{array}{ccc}
J_{11} & J_{12} & J_{13} \\
J_{21} & J_{22} & J_{23} \\
J_{31} & J_{32} & J_{33}
\end{array}\right) .
$$

In our case, the elements of the observed information matrix are derived as $J_{13}=J_{31}=$ $J_{23}=J_{32}=0, J_{33}=m / \theta^{2}$,

$$
\begin{gathered}
J_{11}=n m\left(\frac{1}{\alpha^{2}}-\frac{1}{(\beta-\alpha)^{2}}\right)+\sum_{i=1}^{m} \sum_{j=1}^{n} \frac{z_{i j}^{2} e^{-z_{i j}(\beta-\alpha)}}{\left(1-e^{-z_{i j}(\beta-\alpha)}\right)^{2}}, \\
J_{12}=J_{21}=\frac{n m}{(\beta-\alpha)^{2}}-\sum_{i=1}^{m} \sum_{j=1}^{n} \frac{z_{i j}^{2} e^{-z_{i j}(\beta-\alpha)}}{\left(1-e^{-z_{i j}(\beta-\alpha)}\right)^{2}},
\end{gathered}
$$

and

$$
J_{22}=n m\left(\frac{1}{\beta^{2}}-\frac{1}{(\beta-\alpha)^{2}}\right)+\sum_{i=1}^{m} \sum_{j=1}^{n} \frac{z_{i j}^{2} e^{-z_{i j}(\beta-\alpha)}}{\left(1-e^{-z_{i j}(\beta-\alpha)}\right)^{2}} .
$$

The expectation of the observed information matrix $I(\tau)=E(J(\tau))$ cannot be obtained analytically. It can be evaluated by using numerical integration methods. Then, $\widehat{R}_{C o m p}^{M L E}$ is asymptotically normal with mean $R_{C o m p}$ and asymptotic variance

$$
\sigma_{R_{\text {Comp }}}^{2}=\sum_{j=1}^{3} \sum_{i=1}^{3} \frac{\partial R_{\text {Comp }}}{\partial \tau_{i}} \frac{\partial R_{\text {Comp }}}{\partial \tau_{j}} I_{i j}^{-1},
$$

where $I_{i j}^{-1}$ is the $(i, j)^{t h}$ element of the inverse of $I(\tau)$, see [35]. Afterwards,

$$
\sigma_{R_{\text {Comp }}}^{2}=\left(\frac{\partial R_{\text {Comp }}}{\partial \alpha}\right)^{2} I_{11}^{-1}+2 \frac{\partial R_{\text {Comp }}}{\partial \alpha} \frac{\partial R_{\text {Comp }}}{\partial \beta} I_{12}^{-1}+\left(\frac{\partial R_{\text {Comp }}}{\partial \beta}\right)^{2} I_{22}^{-1}+\left(\frac{\partial R_{\text {Comp }}}{\partial \theta}\right)^{2} I_{33}^{-1},
$$

Note that $I(\tau)$ can be replaced by $J(\tau)$ when $I(\tau)$ is not available in closed form. Therefore, an asymptotic $100(1-\gamma) \%$ confidence interval of $R_{C o m p}$ is obtained as $\left(\widehat{R}_{\text {Comp }}^{M L E} \pm\right.$ $z_{\gamma / 2} \widehat{\sigma}_{R_{\text {Comp }}}$ ), where $z_{\gamma / 2}$ is the upper $\gamma / 2$ th quantile of the standard normal distribution and $\widehat{\sigma}_{R_{\text {Comp }}}$ is the value of $\sigma_{R_{\text {Comp }}}$ at the MLE of the unknown parameters.

\subsection{Nonparametric estimation of $R_{C o m p}$}

In this subsection, we consider the nonparametric estimator of $R_{C o m p}$ based on the strength and stress data.

Let $X_{1}, \ldots, X_{n_{1}}$ and $Y_{1}, \ldots, Y_{n_{2}}$ be two independent random samples from the distributions of $X$ and $Y$, respectively. Birnbaum and McCarty [9] obtained the nonparametric estimate of the simple stress-strength reliability $R=P(X<Y)$ using the Mann-Whitney 
U statistic where $X$ and $Y$ denote the stress and strength variables. This estimate is given by

$$
\widetilde{R}=\frac{1}{n_{1} n_{2}} \sum_{i=1}^{n_{1}} \sum_{j=1}^{n_{2}} I\left(X_{i}<Y_{j}\right)
$$

where $I\left(X_{i}<Y_{j}\right)=1$ if $X_{i}<Y_{j}$ and 0 otherwise. It is known that $\widetilde{R}$ is a consistent, asymptotically normal and minimum variance estimate of $R$, see [3]. Nonparametric estimates of the simple stress-strength reliability and multicomponent reliability were considered by several authors in the literature. Different nonparametric estimates were introduced in this regard. For more details about these estimates, we refer [5,31,32].

In our case, $R_{C o m p}=P\left(Z_{(1)}>T\right)$ can be considered as the simple stress-strength. Based on our strength $Z_{i 1}, \ldots, Z_{i n}, i=1, \ldots m$ and stress $T_{i}, i=1, \ldots, m$ samples, the nonparametric estimate of $R_{C o m p}$, i.e. $\widetilde{R}_{C o m p}$, is given by

$$
\widetilde{R}_{\text {comp }}=\frac{1}{m^{2}} \sum_{i=1}^{m} \sum_{j=1}^{m} I\left(T_{j}<Z_{i(1)}\right),
$$

using $\widetilde{R}$ in Equation (3.6) where $Z_{i(1)}=\min \left(Z_{i 1}, \ldots, Z_{i n}\right), i=1, \ldots m$.

\subsection{Bayesian estimation of $R_{C o m p}$}

In this subsection, we consider Bayesian point and interval estimations of $R_{C o m p}$. In the Bayesian approach, it is assumed that we have prior information about the unknown parameters. Suppose that $\alpha, \beta$ and $\theta$ have independent gamma priors with parameters $\left(a_{i}, b_{i}\right), i=1,2,3$, respectively. The pdf of a gamma random variable $X$ with parameters $(a, b)$ is given by

$$
f(x)=\frac{b^{a}}{\Gamma(a)} x^{a-1} e^{-x b}, \quad x>0, \quad a, b>0,
$$

and denoted by $\operatorname{Gamma}(a, b)$. The joint posterior density function of $\alpha, \beta$ and $\theta$ is given by

$$
\pi(\alpha, \beta, \theta \mid \mathbf{z}, \mathbf{t})=I(\mathbf{z}, \mathbf{t}) \alpha^{n m+a_{1}-1} \beta^{n m+a_{2}-1}(\beta-\alpha)^{-n m} \theta^{a_{3}+m-1} e^{-\alpha b_{1}-\beta b_{2}-\theta\left(b_{3}+\sum_{i=1}^{m} t_{i}\right)} z_{\alpha, \beta},
$$

where $I(\mathbf{z}, \mathbf{t})$ is the normalizing constant

$$
\frac{I(\mathbf{z}, \mathbf{t})^{-1}}{\Gamma\left(a_{3}+m\right)}\left(b_{3}+\sum_{i=1}^{m} t_{i}\right)^{a_{3}+m}=\int_{0}^{\infty} \int_{0}^{\infty}\left(\frac{\alpha \beta}{\beta-\alpha}\right)^{n m} \alpha^{a_{1}-1} \beta^{a_{2}-1} e^{-\alpha b_{1}-\beta b_{2}} z_{\alpha, \beta} d \alpha d \beta
$$

and

$$
z_{\alpha, \beta}=\exp \left(\sum_{i=1}^{m} \sum_{j=1}^{n} \ln \left(e^{-\alpha z_{i j}}-e^{-\beta z_{i j}}\right)\right) .
$$

Bayes estimate of $R_{\text {Comp }}$, i.e. $\widehat{R}_{\text {Comp }}^{\text {Bayes }}$, under the squared error (SE) loss function is

$$
\widehat{R}_{\text {Comp }}^{\text {Bayes }}=\int_{0}^{\infty} \int_{0}^{\infty} \int_{0}^{\infty} R_{\text {Comp }} \pi(\alpha, \beta, \theta \mid \mathbf{z}, \mathbf{t}) d \alpha d \beta d \theta .
$$

Since the above integral is not computed analytically, some approximation methods are required to obtain approximate Bayes estimate. In the next part, we consider the Lindley's approximation and MCMC methods. 
3.4.1. Lindley's approximation. Lindley proposed an approximate method in order to obtain a numerical result for the computation of the ratio of two integrals in [29]. This procedure, applied to the posterior expectation of the function $u(\theta)$ for a given $\mathbf{x}$ is

$$
E(u(\theta) \mid \mathbf{x})=\frac{\int u(\theta) e^{Q(\theta)} d \lambda}{\int e^{Q(\theta)} d \lambda}
$$

where $Q(\theta)=l(\theta)+\rho(\theta), l(\theta)$ is the logarithm of the likelihood function and $\rho(\theta)$ is the logarithm of the prior density of $\theta$. Using Lindley's approximation, $E(u(\theta) \mid \mathbf{x})$ is approximately estimated by

$$
\begin{aligned}
E(u(\theta) \mid \mathbf{x})= & {\left[u+\frac{1}{2} \sum_{i} \sum_{j}\left(u_{i j}+2 u_{i} \rho_{j}\right) \sigma_{i j}+\frac{1}{2} \sum_{i} \sum_{j} \sum_{k} \sum_{l} L_{i j k} \sigma_{i j} \sigma_{k l} u_{l}\right]_{\widehat{\lambda}} } \\
& + \text { terms of order } n^{-2} \text { or smaller, }
\end{aligned}
$$

where $\theta=\left(\theta_{1}, \theta_{2}, \ldots, \theta_{m}\right), i, j, k, l=1, \ldots, m, \widehat{\theta}$ is the MLE of $\theta, u=u(\theta), u_{i}=\partial u / \partial \theta_{i}$, $u_{i j}=\partial^{2} u / \partial \theta_{i} \partial \theta_{j}, L_{i j}=\partial^{2} l / \partial \theta_{i} \partial \theta_{j}, L_{i j k}=\partial^{3} l / \partial \theta_{i} \partial \theta_{j} \partial \theta_{k}, \rho_{j}=\partial \rho / \partial \theta_{j}$, and $\sigma_{i j}=(i, j)$ th element in the inverse of the matrix $\left\{-L_{i j}\right\}$ all evaluated at the MLE of the parameters.

For the three parameter case $\theta=\left(\theta_{1}, \theta_{2}, \theta_{3}\right)$, Lindley's approximation gives the approximate Bayes estimate as

$$
\begin{aligned}
\widehat{u}_{B} & =E(u(\theta) \mid \mathbf{x})=u+\left(u_{1} a_{1}+u_{2} a_{2}+u_{3} a_{3}+a_{4}+a_{5}\right)+0.5\left[A \left(u_{1} \sigma_{11}+u_{2} \sigma_{12}\right.\right. \\
& \left.\left.+u_{3} \sigma_{13}\right)+B\left(u_{1} \sigma_{21}+u_{2} \sigma_{22}+u_{3} \sigma_{23}\right)+C\left(u_{1} \sigma_{31}+u_{2} \sigma_{32}+u_{3} \sigma_{33}\right)\right],
\end{aligned}
$$

evaluated at $\widehat{\theta}=\left(\widehat{\theta}_{1}, \widehat{\theta}_{2}, \widehat{\theta}_{3}\right)$, where

$$
\begin{gathered}
a_{i}=\rho_{1} \sigma_{i 1}+\rho_{2} \sigma_{i 2}+\rho_{3} \sigma_{i 3}, \quad i=1,2,3, \\
a_{4}=u_{12} \sigma_{12}+u_{13} \sigma_{13}+u_{23} \sigma_{23}, a_{5}=0.5\left(u_{11} \sigma_{11}+u_{22} \sigma_{22}+u_{33} \sigma_{33}\right), \\
A=\sigma_{11} L_{111}+2 \sigma_{12} L_{121}+2 \sigma_{13} L_{131}+2 \sigma_{23} L_{231}+\sigma_{22} L_{221}+\sigma_{33} L_{331}, \\
B=\sigma_{11} L_{112}+2 \sigma_{12} L_{122}+2 \sigma_{13} L_{132}+2 \sigma_{23} L_{232}+\sigma_{22} L_{222}+\sigma_{33} L_{332}, \\
C=\sigma_{11} L_{113}+2 \sigma_{12} L_{123}+2 \sigma_{13} L_{133}+2 \sigma_{23} L_{233}+\sigma_{22} L_{223}+\sigma_{33} L_{333} .
\end{gathered}
$$

In our case, $\left(\theta_{1}, \theta_{2}, \theta_{3}\right) \equiv(\alpha, \beta, \theta)$, and $u \equiv u(\alpha, \beta, \theta)=R_{\text {Comp }}$ from Equation (2.3). First, $\sigma_{i j}, i, j=1,2,3$ are computed by using the partial derivatives $L_{i j}=-J_{i j}, i, j=$ $1,2,3$. Second, we evaluate the constants as $\rho_{1}=\left(\left(a_{1}-1\right) / \alpha\right)-1, \rho_{2}=\left(\left(a_{2}-1\right) / \beta\right)-b_{2}$ and $\rho_{3}=\left(\left(a_{3}-1\right) / \theta\right)-b_{3}$ using the logarithm of the prior density, and $L_{333}=2 \mathrm{~m} / \theta$,

$$
\begin{aligned}
& L_{111}=2 n m\left(\frac{1}{\alpha^{3}}+\frac{1}{(\beta-\alpha)^{3}}\right)-\sum_{i=1}^{m} \sum_{j=1}^{n} \frac{z_{i j}^{3} e^{-z_{i j}(\beta-\alpha)}\left(1+e^{-z_{i j}(\beta-\alpha)}\right)}{\left(1-e^{-z_{i j}(\beta-\alpha)}\right)^{3}}, \\
& L_{121}=L_{112}=-2 n m \frac{1}{(\beta-\alpha)^{3}}+\sum_{i=1}^{m} \sum_{j=1}^{n} \frac{z_{i j}^{3} e^{-z_{i j}(\beta-\alpha)}\left(1+e^{-z_{i j}(\beta-\alpha)}\right)}{\left(1-e^{-z_{i j}(\beta-\alpha)}\right)^{3}}, \\
& L_{122}=L_{221}=2 n m \frac{1}{(\beta-\alpha)^{3}}-\sum_{i=1}^{m} \sum_{j=1}^{n} \frac{z_{i j}^{3} e^{-z_{i j}(\beta-\alpha)}\left(1+e^{-z_{i j}(\beta-\alpha)}\right)}{\left(1-e^{-z_{i j}(\beta-\alpha)}\right)^{3}}, \\
& L_{222}=2 n m\left(\frac{1}{\beta^{3}}-\frac{1}{(\beta-\alpha)^{3}}\right)+\sum_{i=1}^{m} \sum_{j=1}^{n} \frac{z_{i j}^{3} e^{-z_{i j}(\beta-\alpha)}\left(1+e^{-z_{i j}(\beta-\alpha)}\right)}{\left(1-e^{-z_{i j}(\beta-\alpha)}\right)^{3}} .
\end{aligned}
$$


$u_{i j}, i, j=1,2,3$ are computed by taking the partial derivative of $R_{C o m p}$ from Equation (2.3). Lastly, we obtain $A=\sigma_{11} L_{111}+2 \sigma_{12} L_{121}+\sigma_{22} L_{221}, B=\sigma_{11} L_{112}+2 \sigma_{12} L_{122}+$ $\sigma_{22} L_{222}$, and $C=\sigma_{33} L_{333}$. Then, the approximate Bayes estimator of $R_{C o m p}$ is given by

$$
\begin{aligned}
\widehat{R}_{\text {Comp }}^{\text {Lindley }} & =R_{\text {Comp }}+\left[u_{1} a_{1}+u_{2} a_{2}+u_{3} a_{3}+a_{4}+a_{5}\right]+\frac{1}{2}\left[A\left(u_{1} \sigma_{11}+u_{2} \sigma_{12}+u_{3} \sigma_{13}\right)\right. \\
& \left.+B\left(u_{1} \sigma_{21}+u_{2} \sigma_{22}+u_{3} \sigma_{23}\right)+C\left(u_{1} \sigma_{31}+u_{2} \sigma_{32}+u_{3} \sigma_{33}\right)\right],
\end{aligned}
$$

where all the parameters are evaluated at MLEs $(\widehat{\alpha}, \widehat{\beta}, \widehat{\theta})$.

3.4.2. MCMC method. The joint posterior density function of $\alpha, \beta$ and $\theta$ given data is stated in Equation (3.8). Then, the marginal posterior density functions of the parameters are given respectively as

$$
\begin{gathered}
\theta \mid \mathbf{t} \sim \operatorname{Gamma}\left(m+a_{3}, b_{3}+\sum_{i=1}^{m} t_{i}\right), \\
\pi(\alpha \mid \beta, \mathbf{z}) \propto \alpha^{n m+a_{1}-1}(\beta-\alpha)^{-n m} e^{-\alpha b_{1}} \exp \left(\sum_{i=1}^{m} \sum_{j=1}^{n} \ln \left(e^{-\alpha z_{i j}}-e^{-\beta z_{i j}}\right)\right),
\end{gathered}
$$

and

$$
\pi(\beta \mid \alpha, \mathbf{z}) \propto \beta^{n m+a_{2}-1}(\beta-\alpha)^{-n m} e^{-\beta b_{2}} \exp \left(\sum_{i=1}^{m} \sum_{j=1}^{n} \ln \left(e^{-\alpha z_{i j}}-e^{-\beta z_{i j}}\right)\right) .
$$

Hence, samples of $\theta$ can be readily generated by using a gamma distribution. Since the marginal posterior distributions of $\alpha$ and $\beta$ are not a well-known distribution, it is not possible to generate sample directly by standard methods. Therefore, we use hybrid Metropolis-Hastings and Gibbs sampling algorithm to generate samples from $\pi(\alpha \mid \beta, \mathbf{z})$ and $\pi(\beta \mid \alpha, \mathbf{z})$, (for more details see $[18,39]$ ).

Step 1: Start with initial guess $\alpha^{(0)}, \beta^{(0)}$.

Step 2: Set $i=1$.

Step 3: Generate $\theta^{(i)}$ from $\operatorname{Gamma}\left(m+a_{3}, b_{3}+\sum_{i=1}^{m} t_{i}\right)$.

Step 4: Generate $\alpha^{(i)}$ from $\pi(\alpha \mid \beta, \mathbf{z})$ using the Metropolis-Hastings algorithm with the proposal distribution $q_{1}(\alpha) \equiv N\left(\alpha^{(i-1)}, 1\right)$ as follows.

(a) Let $v=\alpha^{(i-1)}$.

(b) Generate $w$ from the proposal distribution $q$.

(c) Let $p(v, w)=\min \left\{1, \frac{\pi\left(w \mid \beta^{(i-1)}, \mathbf{z}\right) q(v)}{\pi\left(v \mid \beta^{(i-1)}, \mathbf{z}\right) q(w)}\right\}$.

(d) Generate $u$ from Uniform $(0,1)$. If $u \leq p(v, w)$, then accept the proposal and set $\alpha^{(i)}=w$; otherwise, set $\alpha^{(i)}=v$.

Step 5: Similarly, $\beta^{(i)}$ is generated from $\pi(\beta \mid \alpha, \mathbf{z})$ using the Metropolis-Hastings algorithm with the proposal distribution $q_{2}(\beta) \equiv N\left(\beta^{(i-1)}, 1\right)$.

Step 6: Compute the $R_{\text {Comp }}^{(i)}$ at $\left(\alpha^{(i)}, \beta^{(i)}, \theta^{(i)}\right)$.

Step 7: Set $i=i+1$.

Step 8: Repeat Steps 2-7, $N$ times and obtain the posterior sample $R_{C o m p}^{(i)}, i=1, \ldots, N$.

This sample is used to compute Bayes estimate and to construct the HPD credible interval for $R_{\text {Comp }}$. Bayes estimate of $R_{\text {Comp }}$ under a $\mathrm{SE}$ loss function is given by

$$
\widehat{R}_{\text {Comp }}^{M C M C}=\frac{1}{N-M} \sum_{i=M+1}^{N-M} R_{C o m p}^{(i)},
$$

where $M$ is the burn-in period. The HPD $100(1-\gamma) \%$ credible interval of $R_{C o m p}$ is obtained by the method of [14]. 


\subsection{Inference on $\Phi_{C o m p}$}

The MRS of our series system under the stress $T$ is the expected remaining strength, and defined by the following conditional expectation $\Phi_{C o m p}=E\left(Z_{(1)}-T \mid Z_{(1)}>T\right)$. The cdf of the conditional random variable $\psi \equiv\left(Z_{(1)}-T \mid Z_{(1)}>T\right)$ is

$$
F_{\psi}(x)=P\left(Z_{(1)}-T \leq x \mid Z_{(1)}>T\right)=\frac{P\left(Z_{(1)} \leq T+x, Z_{(1)}>T\right)}{R_{C o m p}} .
$$

Then, conditioning on $T=t$, we have

$$
\begin{aligned}
P\left(Z_{(1)}\right. & \left.\leq T+x, Z_{(1)}>T\right)=\int_{0}^{\infty} P\left(t<Z_{(1)} \leq t+x\right) d F_{T}(t) \\
& =\int_{0}^{\infty}\left[F_{Z_{(1)}}(t+x)-F_{Z_{(1)}}(t)\right] d F_{T}(t) \\
& =\int_{0}^{\infty}\left[\frac{\left(\beta e^{-\alpha t}-\alpha e^{-\beta t}\right)^{n}}{(\beta-\alpha)^{n}}-\frac{\left(\beta e^{-\alpha(t+x)}-\alpha e^{-\beta(t+x)}\right)^{n}}{(\beta-\alpha)^{n}}\right] \theta e^{-\theta t} d t \\
& \equiv \frac{I_{1}-I_{2}}{(\beta-\alpha)^{n}},
\end{aligned}
$$

for $\alpha \neq \beta$. After some computations, we obtain

$$
I_{1}=\sum_{i=0}^{n}\left(\begin{array}{c}
n \\
i
\end{array}\right)(-1)^{i} \frac{\alpha^{i} \beta^{n-i} \theta}{[\alpha(n-i)+\beta i+\theta]}
$$

and

$$
I_{2}=e^{-\alpha n x} \sum_{i=0}^{n}\left(\begin{array}{c}
n \\
i
\end{array}\right)(-1)^{i} e^{-i x(\beta-\alpha)} \frac{\alpha^{i} \beta^{n-i} \theta}{[\alpha(n-i)+\beta i+\theta]} .
$$

Hence, the cdf and pdf of $\psi$ are given by

$$
F_{\psi}(x)=\frac{1}{R_{C o m p}(\beta-\alpha)^{n}} \sum_{i=0}^{n}\left(\begin{array}{c}
n \\
i
\end{array}\right)(-1)^{i} \frac{\alpha^{i} \beta^{n-i} \theta\left(1-e^{-x(\alpha(n-i)+\beta i)}\right)}{[\alpha(n-i)+\beta i+\theta]},
$$

and

$$
f_{\psi}(x)=\frac{1}{R_{C o m p}(\beta-\alpha)^{n}} \sum_{i=0}^{n}\left(\begin{array}{c}
n \\
i
\end{array}\right)(-1)^{i} \frac{\alpha^{i} \beta^{n-i} \theta[\alpha(n-i)+\beta i] e^{-x(\alpha(n-i)+\beta i)}}{[\alpha(n-i)+\beta i+\theta]},
$$

for $\alpha \neq \beta$. Therefore, the MRS of the system is obtained as

$$
\begin{aligned}
\Phi_{C o m p} & =E(\psi)=\int_{0}^{\infty} x f_{\psi}(x) d x \\
& =\frac{1}{R_{C o m p}(\beta-\alpha)^{n}} \sum_{i=0}^{n}\left(\begin{array}{c}
n \\
i
\end{array}\right)(-1)^{i} \frac{\alpha^{i} \beta^{n-i} \theta}{[\alpha(n-i)+\beta i][\alpha(n-i)+\beta i+\theta]},
\end{aligned}
$$

for $\alpha \neq \beta$. Moreover, $\Phi_{\text {Comp }}$ can be easily derived as

$$
\Phi_{C o m p}=\frac{1}{R_{C o m p}} \sum_{i=0}^{n} \sum_{j=0}^{i}\left(\begin{array}{c}
n \\
i
\end{array}\right) \Gamma(i+1) \frac{\alpha^{j-1} \theta}{n^{i-j+1}(\alpha n+\theta)^{j+1}},
$$

for $\alpha=\beta$. 
3.5.1. Estimation of $\Phi_{C o m p}$. The MLE of $\Phi_{C o m p}$, i.e. $\widehat{\Phi}_{C o m p}^{M L E}$, is computed from Equation (3.13) by using the invariance property of MLE. It is clear that $\widehat{\Phi}_{C o m p}^{M L E}$ is asymptotically normal with mean $\Phi_{C o m p}$ and asymptotic variance is computed by using the formula in Equation (3.4). Hence, an asymptotic $100(1-\gamma) \%$ confidence interval for $\Phi_{\text {Comp }}$ is given by $\left(\widehat{\Phi}_{C o m p}^{M L E} \pm z_{\gamma / 2} \widehat{\sigma}_{\Phi_{C o m p}}\right)$ where $\widehat{\sigma}_{\Phi_{C o m p}}$ is the value of $\sigma_{\Phi_{C o m p}}$ at the MLE of the parameters.

Under the setup made in Subsection 3.4, the Bayes estimator of $\Phi_{\text {Comp }}$ under the SE loss function is given by

$$
\widehat{\Phi}_{\text {Comp }}^{\text {Bayes }}=\int_{0}^{\infty} \int_{0}^{\infty} \int_{0}^{\infty} \Phi_{\text {Comp }} \pi(\alpha, \beta, \theta \mid \mathbf{z}, \mathbf{t}) d \alpha d \beta d \theta .
$$

Similar to the reliability case, since the above integral cannot be computed analytically, the Bayes estimate of $\Phi_{C o m p}$ under SE loss function is obtained by using Lindley's approximation. It is omitted because of a similar procedure is mentioned in the reliability case.

\section{Estimation of $R_{\text {System }}$ and $\Phi_{\text {System }}$}

In this section, point and interval estimations of the stress-strength reliability and MRS of the series system with cold standby at system level are investigated.

\subsection{MLE of $R_{\text {System }}$}

Suppose that $m$ systems are put on a test each with $n$ original components with $n$ cold standby components at system level in the series system. In this case, the strength data is represented as $Z_{(1), i}, i=1, \ldots, m$ and stress is $T_{i}, i=1, \ldots, m$. Here, $Z_{(1), i}=X_{(1), i}+Y_{(1), i}$ is the lifetime of $i^{\text {th }}$ system as in Section 2. Its pdf and cdf are obtained as in the Equations (2.4) and (2.5), and the pdf of $Z_{(1), i}$ is denoted by $f_{Z_{(1), i}}\left(z_{(1), i}\right), i=1, \ldots, m$. Then, the likelihood function of $\alpha, \beta$ and $\theta$ for the observed sample is

$$
\begin{aligned}
L(\alpha, \beta, \theta \mid \underline{z}, \underline{t}) & =\prod_{i=1}^{m} f_{Z_{(1), i}}\left(z_{(1), i}\right) f_{T}\left(t_{i}\right) \\
& =\left(\frac{n \alpha \beta \theta}{\alpha-\beta}\right)^{m} \prod_{i=1}^{m}\left(e^{-\beta n z_{(1), i}}-e^{-\alpha n z_{(1), i}}\right) e^{-\theta t_{i}} .
\end{aligned}
$$

The corresponding log-likelihood function is

$$
\ell(\alpha, \beta, \theta \mid \underline{z}, \underline{t})=m[\ln (n \alpha \beta \theta)-\ln (\alpha-\beta)]+\sum_{i=1}^{m} \ln \left(e^{-\beta n z_{(1), i}}-e^{-\alpha n z_{(1), i}}\right)-\theta \sum_{i=1}^{m} t_{i} .
$$

By partially differentiating Equation (4.1) with respect to $\alpha$ and $\beta$, we obtain the following non-linear equations

$$
\begin{aligned}
& \frac{\partial \ell}{\partial \alpha}=m\left(\frac{1}{\alpha}+\frac{1}{\beta-\alpha}\right)-\sum_{i=1}^{m} \frac{n z_{(1), i}}{1-e^{-(\beta-\alpha) n z_{(1), i}}}=0, \\
& \frac{\partial \ell}{\partial \beta}=m\left(\frac{1}{\beta}-\frac{1}{\beta-\alpha}\right)+\sum_{i=1}^{m} \frac{n z_{(1), i}}{e^{-(\alpha-\beta) n z_{(1), i}}-1}=0 .
\end{aligned}
$$

The MLEs $\widehat{\alpha}$ and $\widehat{\beta}$ can be obtained by solving the non-linear equations in (4.2) and (4.3) simultaneously. This non-linear equation system can be solved by using numerical methods such as Newton-Raphson and Broyden's methods. The MLE of $\theta$ is derived as $\widehat{\theta}=m / \sum_{i=1}^{m} t_{i}$. After obtaining $\widehat{\alpha}, \widehat{\beta}$ and $\widehat{\theta}$, the MLE of $R_{\text {System }}$, i.e. $\widehat{R}_{\text {System }}^{M L E}$, is given by

$$
\widehat{R}_{\text {System }}^{M L E}=\frac{\widehat{\theta}[\widehat{\theta}+n(\widehat{\alpha}+\widehat{\beta})]}{(\widehat{\beta} n+\widehat{\theta})(\widehat{\alpha} n+\widehat{\theta})},
$$


from Equation (2.6) using the invariance property of MLE.

\subsection{Asymptotic confidence interval of $R_{\text {System }}$}

The elements of the observed information matrix $J(\tau)$, in which $\tau=(\alpha, \beta, \theta)$ and $J(\tau)=\left[J_{i j}\right]=\left[-\partial^{2} \ell / \partial \tau_{i} \partial \tau_{j}\right]$, are derived as $J_{13}=J_{31}=J_{23}=J_{32}=0, J_{33}=m / \theta^{2}$,

$$
\begin{gathered}
J_{11}=m\left(\frac{1}{\alpha^{2}}-\frac{1}{(\beta-\alpha)^{2}}\right)+\sum_{i=1}^{m} \frac{n^{2} z_{(1), i}^{2} e^{-(\beta-\alpha) n z_{(1), i}}}{\left(1-e^{\left.-(\beta-\alpha) n z_{(1), i}\right)^{2}}\right.}, \\
J_{12}=J_{21}=\frac{m}{(\beta-\alpha)^{2}}-\sum_{i=1}^{m} \frac{n^{2} z_{(1), i}^{2} e^{-(\beta-\alpha) n z_{(1), i}}}{\left(1-e^{\left.-(\beta-\alpha) n z_{(1), i}\right)^{2}}\right.},
\end{gathered}
$$

and

$$
J_{22}=m\left(\frac{1}{\beta^{2}}-\frac{1}{(\beta-\alpha)^{2}}\right)+\sum_{i=1}^{m} \frac{n^{2} z_{(1), i}^{2} e^{-(\alpha-\beta) n z_{(1), i}}}{\left(e^{-(\alpha-\beta) n z_{(1), i}}-1\right)^{2}} .
$$

Since the Fisher information matrix $E(J(\tau))$ cannot be obtained analytically, it is computed by applying numerical integration methods. Then, $\widehat{R}_{\text {System }}^{M L E}$ is asymptotically normal with mean $R_{\text {System }}$ and asymptotic variance is computed by using the formula in Equation (3.4). Hence, an asymptotic $100(1-\gamma) \%$ confidence interval of $R_{\text {System }}$ is given by $\left(\widehat{R}_{\text {System }}^{M L E} \pm z_{\gamma / 2} \widehat{\sigma}_{R_{\text {System }}}\right)$ where $z_{\gamma / 2}$ is the upper $\gamma / 2$ th quantile of the standard normal distribution and $\widehat{\sigma}_{R_{\text {System }}}$ is the value of $\sigma_{R_{\text {System }}}$ at the MLE of the parameters.

\subsection{Nonparametric estimation of $R_{\text {System }}$}

In this case, the nonparametric estimate of $R_{\text {System }}=P\left(Z_{(1)}>T\right)$ is given by

$$
\widetilde{R}_{\text {System }}=\frac{1}{m^{2}} \sum_{i=1}^{m} \sum_{j=1}^{m} I\left(T_{j}<Z_{(1), i}\right)
$$

using the similar way in Subsection 3.3 based on the samples $Z_{(1), i}$ and $T_{i}, i=1, \ldots, m$ where $Z_{(1), i}=X_{(1), i}+Y_{(1), i}, i=1, \ldots, m$.

\subsection{Bayesian estimation of $R_{\text {System }}$}

In Bayesian case, it is assumed that $\alpha, \beta$ and $\theta$ have independent gamma priors with parameters $\left(a_{i}, b_{i}\right), i=1,2,3$, respectively. Then, the joint posterior density function of $\alpha, \beta$ and $\theta$ is

$$
\begin{aligned}
\pi(\alpha, \beta, \theta \mid \mathbf{z}, \mathbf{t}) \propto & \alpha^{m+a_{1}-1} \beta^{m+a_{2}-1}(\beta-\alpha)^{-m} \theta^{m+a_{3}-1} e^{-\alpha b_{1}-\beta b_{2}-\theta\left(b_{3}+\sum_{i=1}^{m} t_{i}\right)} \\
& \cdot \exp \left(\sum_{i=1}^{m} \ln \left(e^{-\alpha n z_{(1), i}}-e^{-\beta n z_{(1), i}}\right)\right) .
\end{aligned}
$$

Bayes estimate of $R_{\text {System }}$, i.e. $\widehat{R}_{\text {System }}^{\text {Bayes }}$, under the SE loss function is

$$
\widehat{R}_{\text {System }}^{\text {Bayes }}=\int_{0}^{\infty} \int_{0}^{\infty} \int_{0}^{\infty} R_{\text {System }} \pi(\alpha, \beta, \theta \mid \mathbf{z}, \mathbf{t}) d \alpha d \beta d \theta .
$$

Since the $\widehat{R}_{\text {System }}^{\text {Bayes }}$ cannot be computed analytically, we resort to MCMC methods. 
4.4.1. MCMC method. The joint posterior density function of $\alpha, \beta$ and $\theta$ given data is stated in Equation (4.5). Then, the marginal posterior density functions of the parameters are given respectively as

$$
\begin{gathered}
\theta \mid \mathbf{t} \sim \operatorname{Gamma}\left(m+a_{3}, b_{3}+\sum_{i=1}^{m} t_{i}\right), \\
\pi(\alpha \mid \beta, \mathbf{z}) \propto \alpha^{m+a_{1}-1}(\beta-\alpha)^{-m} e^{-\alpha b_{1}} \exp \left(\sum_{i=1}^{m} \ln \left(e^{-\alpha n z_{(1), i}}-e^{-\beta n z_{(1), i}}\right)\right),
\end{gathered}
$$

and

$$
\pi(\beta \mid \alpha, \mathbf{z}) \propto \beta^{m+a_{2}-1}(\beta-\alpha)^{-m} e^{-\beta b_{2}} \exp \left(\sum_{i=1}^{m} \ln \left(e^{-\alpha n z_{(1), i}}-e^{-\beta n z_{(1), i}}\right)\right) .
$$

Therefore, samples of $\theta$ can be easily generated by using a gamma distribution. Since the marginal posterior distributions of $\alpha$ and $\beta$ are not well-known distributions, it is not possible to generated sample directly by standard methods. Hence, Bayes estimate and HPD credible interval of $R_{\text {System }}$ are computed by using the hybrid Metropolis-Hastings and Gibbs sampling algorithm. Since this procedure is similar to in Subsection 3.4.2, it is not detailed.

\subsection{Inference on $\Phi_{\text {System }}$}

The MRS of the series system under the stress $T$ is defined by the following conditional expectation $\Phi_{\text {System }}=E\left(Z_{(1)}-T \mid Z_{(1)}>T\right)$. The cdf of the conditional random variable $\psi \equiv\left(Z_{(1)}-T \mid Z_{(1)}>T\right)$ is

$$
F_{\psi}(x)=P\left(Z_{(1)}-T \leq x \mid Z_{(1)}>T\right)=\frac{P\left(Z_{(1)} \leq T+x, Z_{(1)}>T\right)}{R_{\text {System }}} .
$$

Then, conditioning on $T=t$, we have

$$
\begin{aligned}
P\left(Z_{(1)}\right. & \left.\leq T+x, Z_{(1)}>T\right)=\int_{0}^{\infty} P\left(t<Z_{(1)} \leq t+x\right) d F_{T}(t) \\
& =\int_{0}^{\infty}\left[F_{Z_{(1)}}(t+x)-F_{Z_{(1)}}(t)\right] d F_{T}(t) \\
& =\frac{\theta}{(\beta-\alpha)}\left[\frac{\alpha\left(e^{-\beta n x}-1\right)}{\beta n+\theta}-\frac{\beta\left(e^{-\alpha n x}-1\right)}{\alpha n+\theta}\right],
\end{aligned}
$$

for $\alpha \neq \beta$. Hence, the cdf and pdf of $\psi$ are given by

$$
F_{\psi}(x)=\frac{\theta}{R_{\text {System }}(\beta-\alpha)}\left[\frac{\alpha\left(e^{-\beta n x}-1\right)}{\beta n+\theta}-\frac{\beta\left(e^{-\alpha n x}-1\right)}{\alpha n+\theta}\right],
$$

and

$$
f_{\psi}(x)=\frac{\theta \alpha \beta n}{R_{\text {System }}(\beta-\alpha)}\left(\frac{e^{-\alpha n x}}{\alpha n+\theta}-\frac{e^{-\beta n x}}{\beta n+\theta}\right),
$$

for $\alpha \neq \beta$. Therefore, the MRS of our series system is obtained as

$$
\begin{aligned}
\Phi_{\text {System }} & =E(\psi)=\int_{0}^{\infty} x f_{\psi}(x) d x \\
& =\frac{\alpha \beta \theta}{R_{\text {System }}(\beta-\alpha) n}\left[\frac{1}{\alpha^{2}(\alpha n+\theta)}-\frac{1}{\beta^{2}(\beta n+\theta)}\right],
\end{aligned}
$$

for $\alpha \neq \beta$. Moreover, for $\alpha=\beta$, $\Phi_{\text {System }}$ is derived by using $R_{\text {System }}$ in (2.6) as

$$
\Phi_{\text {System }}=\frac{3 \alpha n+\theta}{\alpha n(2 \alpha n+\theta)} .
$$


4.5.1. Estimation of $\Phi_{\text {System }}$. The MLE of $\Phi_{\text {System }}$, i.e. $\widehat{\Phi}_{\text {System }}^{M L E}$, is computed from Equation (4.6) by using the invariance property of MLE. It is clear that $\widehat{\Phi}_{\text {System }}^{M L E}$ is asymptotically normal with mean $\Phi_{\text {System }}$ and asymptotic variance is evaluated by using the formula in Equation (3.4). Then, an asymptotic $100(1-\gamma) \%$ confidence interval of $\Phi_{\text {System }}$ is obtained as $\left(\widehat{\Phi}_{\text {System }}^{M L E} \pm z_{\gamma / 2} \widehat{\sigma}_{\Phi_{\text {System }}}\right)$ where $\widehat{\sigma}_{\Phi_{\text {System }}}$ is the value of $\sigma_{\Phi_{\text {System }}}$ at the MLE of the parameters. Under the setup made in Subsection 4.4, the Bayes estimate of $\Phi_{\text {System }}$ under the SE loss function cannot be computed analytically. Therefore, Bayes estimate and HPD credible interval of $\Phi_{\text {System }}$ are computed by using the hybrid Metropolis-Hastings and Gibbs sampling algorithm as in the reliability case.

\section{Simulation study}

In this section, we perform a Monte Carlo simulation study to compare performance of the all the proposed estimates. The point and interval estimates of $R_{C o m p}, R_{S y s t e m}, \Phi_{C o m p}$ and $\Phi_{\text {System }}$ are computed based on the Monte Carlo simulations. The performances of the point estimates are compared by using mean square error (MSE) and estimated risk (ER) for ML and Bayes estimates, respectively. The ER of $\theta$ for the $\widehat{\theta}$ estimate is given by

$$
E R(\theta)=\frac{1}{N} \sum_{i=1}^{N}\left(\widehat{\theta}_{i}-\theta_{i}\right)^{2}
$$

under the SE loss function. Average length (AL) of the confidence interval and coverage probability $(\mathrm{CP})$ provide a comparison between the interval estimates. The $\mathrm{CP}$ of a confidence interval is the proportion of time that the interval contains the parameter of interest. All results are obtained based on 2500 replications. The simulation studies have been carried out by using MATLAB and statistical software R [34].

In MLE case of the simulation study, we use the Broyden or Newton-Raphson methods for solving the non-linear equations using the nleqslv [21] package in software $\mathrm{R}$.

In Bayesian case of the simulation study, we have selected hyperparameters as $a_{1}=\alpha$, $a_{2}=\beta, a_{3}=\theta, b_{1}=b_{2}=b_{3}=1$ for the informative prior case and $a_{i}=b_{i}=0, i=1,2,3$ for the non-informative prior case. Bayes estimates of the stress-strength reliability and MRS of the systems are computed by using Lindley's approximation and MCMC method. In the MCMC cases, we run two MCMC chains with fairly different initial values and generate 5000 iterations for each chain. In order to reduce the effect of the starting distribution, the first 2500 results of each sequence are discarded, which is called burnin. So as to cut off the dependence between the results in the Markov chain, only every $d^{\text {th }}$ draw of the chain is saved, which is called thinning. In our cases, Bayesian MCMC estimates are evaluated by the means of the every $5^{\text {th }}$ sampled values after the discarding procedure. Moreover, the convergency of the MCMC chains has been monitored by using the scale reduction factor estimate in [18]. The estimate is given by $\sqrt{\operatorname{Var}(\psi) / W}$, where $\psi$ is the estimand of interest, $\operatorname{Var}(\psi)=(n-1) W / n+B / n$ with the iteration number $n$ for each chain, the between-sequence variance $B$ and the within-sequence variance $W$. In the following simulation studies, the scale factor values of all the MCMC estimators are found to be below 1.1. It is an acceptable value for their convergence.

In Tables 1 and 2, we have reported the ML and Bayesian point estimates of $R_{C o m p}$ and corresponding interval estimates for different component $(n)$ and sample $(m)$ sizes. Bayes estimates of $R_{C o m p}$ are obtained by using both Lindley's approximation and MCMC method. The true values of the parameters are taken as $(\alpha, \beta, \theta)=(1.5,4,6)$ and $(5,3,15)$ for $n=3,5,7$ and 2, 4,6, respectively. The results in Table 1 showed that Bayes estimates of $R_{\text {Comp }}$ based on informative prior have the best performance in terms of error ER. We further observe that Bayes estimate using Lindley's approximation provide relatively better results than the MCMC method. In general, we can order the MSE and ER of 
estimates as $E R\left(\widehat{R}_{\text {Comp }}^{\text {Lindley }}\right)<E R\left(\widehat{R}_{\text {Comp }}^{M C M C}\right)<M S E\left(\widehat{R}_{\text {Comp }}\right)$ based on informative prior. We also note that MSE, ER, and bias of the estimates tend to decrease as the sample size increases. Table 2 indicates that the AL of the asymptotic confidence interval is wider than the corresponding HPD interval. However, CPs of the HPD interval is not as close to the nominal value 0.95 as the asymptotic confidence interval.

Table 1. Estimates of $R_{C o m p}$ for $(\alpha, \beta, \theta)=(1.5,4,6)$ and $(5,3,15)$.

\begin{tabular}{|c|c|c|c|c|c|c|c|c|c|c|c|c|c|c|}
\hline & & & \multicolumn{3}{|c|}{$M L E$} & \multicolumn{6}{|c|}{ Bayes (Inf. prior) } & \multicolumn{3}{|c|}{ Bayes (Non-inf. prior). } \\
\hline$n$ & $m$ & $R_{\text {Comp }}$ & $\widehat{R}_{\text {Comp }}$ & Bias & MSE & $\widehat{R}_{\text {Comp }}^{\text {Lindley }}$ & Bias & ER & $\widehat{R}_{C o m p}^{M C M C}$ & Bias & ER & $\widehat{R}_{\text {Comp }}^{\text {Lindley }}$ & Bias & ER \\
\hline \multicolumn{15}{|c|}{$(\alpha, \beta, \theta)=(1.5,4,6)$} \\
\hline \multirow[t]{4}{*}{3} & 10 & 0.81070 & 0.80060 & -0.01011 & 0.00643 & 0.82738 & 0.01667 & 0.00577 & 0.77493 & -0.03577 & 0.00416 & 0.78825 & -0.02245 & 0.01133 \\
\hline & 20 & & 0.80149 & -0.00921 & 0.00352 & 0.81933 & 0.00862 & 0.00273 & 0.78266 & -0.02805 & 0.00283 & 0.79740 & -0.01331 & 0.00528 \\
\hline & 30 & & 0.80264 & -0.00806 & 0.00230 & 0.81373 & 0.00303 & 0.00239 & 0.78807 & -0.02264 & 0.00207 & 0.80134 & -0.00936 & 0.00389 \\
\hline & 40 & & 0.80636 & -0.00435 & 0.00169 & 0.81332 & 0.00261 & 0.00146 & 0.79493 & -0.01578 & 0.00151 & 0.80645 & -0.00426 & 0.00245 \\
\hline \multirow[t]{4}{*}{5} & 10 & 0.73678 & 0.72864 & -0.00812 & 0.00810 & 0.75074 & 0.01396 & 0.00461 & 0.70096 & -0.03582 & 0.00470 & 0.71891 & -0.01786 & 0.01120 \\
\hline & 20 & & 0.73122 & -0.00556 & 0.00452 & .73898 & 0.00220 & 0.00292 & 0.71362 & -0.02316 & 0.00333 & 0.72801 & -0.00877 & 0.00578 \\
\hline & 25 & & 0.73088 & -0.00589 & 0.00342 & 0.73575 & -0.00102 & 0.00244 & 0.71706 & -0.01972 & 0.00266 & 0.728037 & -0.00874 & 0.00472 \\
\hline & 30 & & 0.73212 & -0.00466 & 0.00301 & 0.73538 & -0.00140 & 0.00211 & 0.72085 & -0.01593 & 0.00239 & 0.73041 & -0.00637 & 0.00371 \\
\hline \multirow[t]{4}{*}{7} & 10 & 0.68197 & 0.67817 & -0.00380 & 0.00935 & 0.68675 & 0.00478 & 0.00388 & 0.65023 & -0.03174 & 0.00488 & 0.66882 & -0.01315 & 0.01181 \\
\hline & 15 & & 0.68079 & -0.00118 & 0.00648 & 0.68360 & 0.00163 & 0.00334 & 0.65992 & -0.02205 & 0.00393 & 0.67621 & -0.00575 & 0.00808 \\
\hline & 20 & & 0.68079 & -0.00117 & 0.00477 & 0.68130 & -0.00067 & 0.00293 & 0.66647 & -0.01550 & 0.00321 & 0.67757 & -0.00440 & 0.00595 \\
\hline & 25 & & 0.68107 & -0.00186 & 0.00396 & 0.67865 & -0.00332 & 0.00258 & 0.66798 & -0.01399 & 0.00288 & 0.67702 & -0.00494 & 0.00454 \\
\hline \multicolumn{15}{|c|}{$(\alpha, \beta, \theta)=(5,3,15)$} \\
\hline \multirow[t]{4}{*}{2} & 10 & 0.92298 & 0.92508 & 0.00210 & 0.00130 & 0.94869 & 0.02571 & 0.00128 & 0.90354 & -0.01944 & 0.00085 & 0.92347 & 0.00049 & 0.00219 \\
\hline & 20 & & 0.91514 & -0.00784 & 0.00103 & 0.94305 & 0.02007 & 0.00081 & 0.90287 & -0.02011 & 0.00083 & 0.91793 & -0.00505 & 0.00147 \\
\hline & 30 & & 0.91483 & -0.00815 & 0.00072 & 0.93744 & 0.01446 & 0.00059 & 0.90500 & -0.01798 & 0.00066 & 0.91807 & -0.00491 & 0.00110 \\
\hline & 40 & & 0.91666 & -0.00632 & 0.00050 & 0.93325 & 0.01027 & 0.00043 & 0.90820 & -0.01477 & 0.00049 & 0.92064 & -0.00234 & 0.00079 \\
\hline \multirow[t]{4}{*}{4} & 10 & 0.86536 & 0.85788 & -0.00747 & 0.00374 & 0.88763 & 0.02227 & 0.00248 & 0.83667 & -0.02867 & 0.00179 & 0.85605 & -0.00931 & 0.00554 \\
\hline & 20 & & 0.85573 & -0.00962 & 0.00223 & 0.87780 & 0.01244 & 0.00100 & 0.84288 & -0.02247 & 0.00139 & 0.85868 & -0.00667 & 0.00310 \\
\hline & 30 & & 0.85518 & -0.01017 & 0.00158 & 0.87155 & 0.00620 & 0.00080 & 0.84690 & -0.01845 & 0.00109 & 0.86057 & -0.00478 & 0.00218 \\
\hline & 40 & & 0.85767 & -0.00768 & 0.00115 & 0.86847 & 0.00312 & 0.00074 & 0.85068 & -0.01468 & 0.00084 & 0.86212 & -0.00323 & 0.00164 \\
\hline \multirow[t]{4}{*}{6} & 10 & 0.81959 & 0.80531 & -0.01428 & 0.00563 & 0.83386 & 0.01426 & 0.00306 & 0.78646 & -0.03313 & 0.00239 & 0.80384 & -0.01575 & 0.00765 \\
\hline & 20 & & 0.81071 & -0.00888 & 0.00293 & 0.82620 & 0.00661 & 0.00112 & 0.79933 & -0.02026 & 0.00153 & 0.81554 & -0.00406 & 0.00403 \\
\hline & 25 & & 0.81114 & -0.00845 & 0.00247 & 0.82361 & 0.00401 & 0.00107 & 0.80216 & -0.01743 & 0.00139 & 0.81661 & -0.00299 & 0.00332 \\
\hline & 30 & & 0.81246 & -0.00713 & 0.00198 & 0.82236 & 0.00277 & 0.00106 & 0.80315 & -0.01644 & 0.00137 & 0.81782 & -0.00178 & 0.00274 \\
\hline
\end{tabular}

Table 2. Average lengths and coverage probabilities of $R_{\text {Comp }}$ for $(\alpha, \beta, \theta)=(1.5,4,6)$ and $(5,3,15)$.

\begin{tabular}{ccc|cc|cc|ccc|cc|cc}
\hline & & \multicolumn{2}{|c|}{$A C I$} & \multicolumn{2}{c|}{$H P D$} & & \multicolumn{2}{c|}{$A C I$} & \multicolumn{2}{c}{$H P D$} \\
\hline$n$ & $m$ & $R_{\text {Comp }}$ & $\mathrm{AL}$ & $\mathrm{CP}$ & $\mathrm{AL}$ & $\mathrm{CP}$ & $n$ & $m$ & $R_{\text {Comp }}$ & $\mathrm{AL}$ & $\mathrm{CP}$ & $\mathrm{AL}$ & $\mathrm{CP}$ \\
\hline 3 & 10 & 0.81070 & 0.32606 & 0.9220 & 0.17645 & 0.8740 & 2 & 10 & 0.92298 & 0.17323 & 0.9048 & 0.10566 & 0.9840 \\
& 20 & & 0.23470 & 0.9372 & 0.14606 & 0.8556 & 20 & & 0.13639 & 0.9520 & 0.08727 & 0.9372 \\
& 30 & & 0.19238 & 0.9520 & 0.12798 & 0.8600 & 30 & & 0.11246 & 0.9636 & 0.07693 & 0.9316 \\
& 40 & & 0.16526 & 0.9468 & 0.11430 & 0.8668 & 40 & & 0.09582 & 0.9672 & 0.06842 & 0.9360 \\
\hline 5 & 10 & 0.73678 & 0.36759 & 0.9296 & 0.18997 & 0.8444 & 4 & 10 & 0.86536 & 0.25642 & 0.9148 & 0.13519 & 0.9380 \\
& 20 & & 0.26308 & 0.9344 & 0.15209 & 0.8184 & 20 & & 0.18603 & 0.9468 & 0.10784 & 0.8884 \\
& 25 & & 0.23681 & 0.9456 & 0.14059 & 0.8268 & 30 & & 0.15288 & 0.9472 & 0.09178 & 0.8624 \\
& 30 & & 0.21599 & 0.9408 & 0.13353 & 0.8228 & & 40 & & 0.13120 & 0.9464 & 0.08378 & 0.8668 \\
\hline 7 & 10 & 0.68197 & 0.38374 & 0.9520 & 0.19082 & 0.8380 & 6 & 10 & 0.81959 & 0.30389 & 0.9344 & 0.14710 & 0.9000 \\
& 15 & & 0.31606 & 0.9280 & 0.16546 & 0.8032 & 20 & & 0.21484 & 0.9420 & 0.11246 & 0.8632 \\
& 20 & & 0.27551 & 0.9324 & 0.14867 & 0.8012 & 25 & & 0.19232 & 0.9420 & 0.10379 & 0.8548 \\
& 25 & & 0.24735 & 0.9328 & 0.15226 & 0.8244 & 30 & & 0.17534 & 0.9436 & 0.10582 & 0.8680 \\
\hline
\end{tabular}

When the cold standby is applied at component level, we have encountered convergence problems in the MCMC case for the large values of $n$ and $m$. The $n m$ is seen as a power of some terms in the marginal posterior densities of $\alpha$ and $\beta$ given in Equations (3.10) and 
(3.11). Therefore, this situation causes an indeterminate ratio in the Metropolis-Hastings algorithm. Since the performance of Bayes estimate of $R_{C o m p}$ using Lindley's approximation is generally better than the MCMC method in Table 1, Lindley's approximation can be considered as a good method. That is why Bayes estimates for the large values of $n$ and $m$ are computed by using Lindley's approximation in the Figure 2.
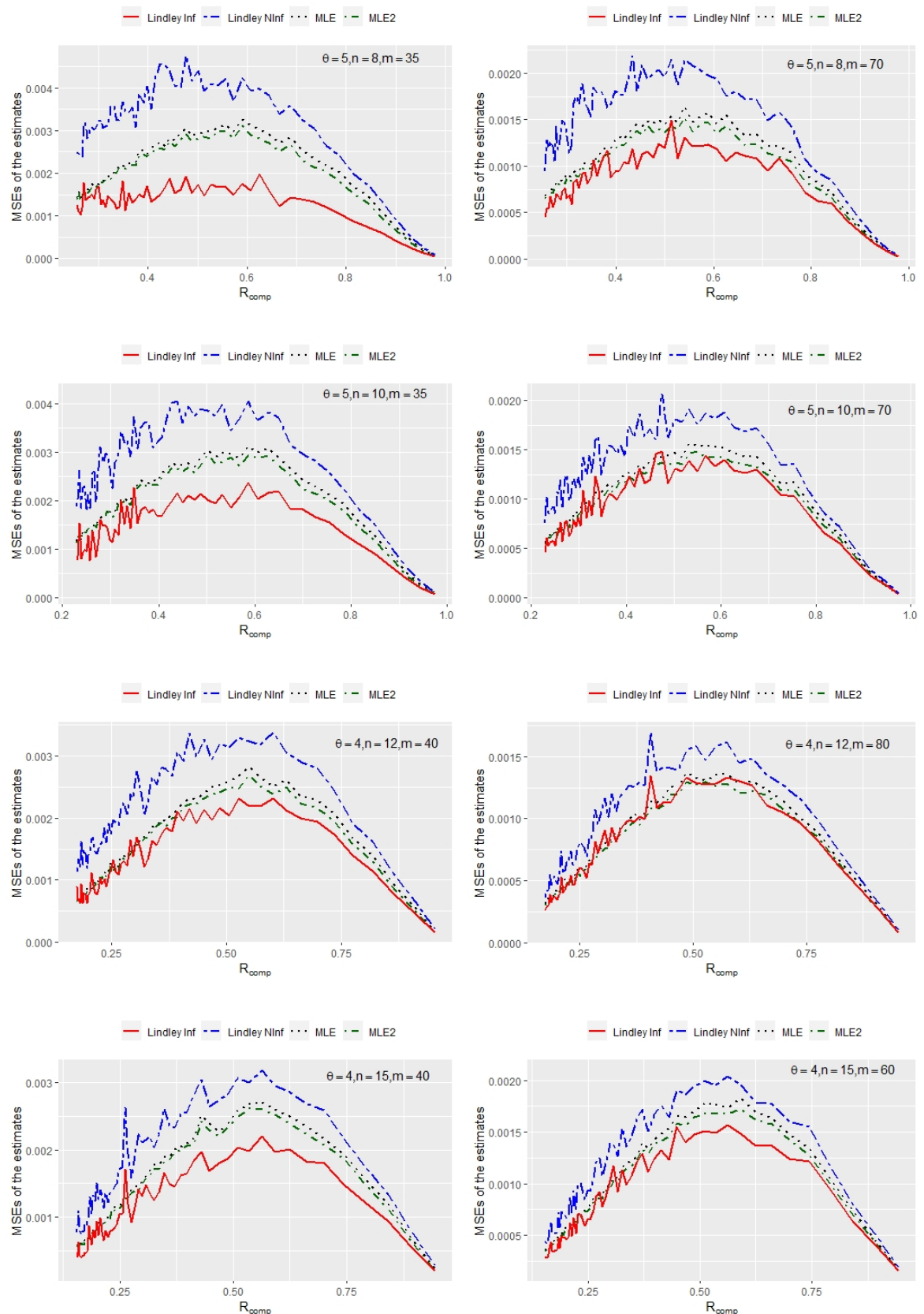

Figure 2. Plots for the $R_{C o m p}$ estimates.

In Figure 2, MSEs of two different ML estimates and ERs of Bayesian estimates based on the informative and non-informative priors are plotted for different parameters and sample sizes. The ML and Bayes estimates of $R_{C o m p}$ are evaluated by using Equations (2.3) and (3.9) at the ML estimates of $(\alpha, \beta, \theta)$ which are obtained from the log-likelihood function in Equation (3.1). Moreover, another ML estimation of $R_{C o m p}$, called MLE2, is evaluated 
at $\widehat{\alpha}_{M L E}=1 / \bar{X}, \widehat{\beta}_{M L E}=1 / \bar{Y}$ and $\widehat{\theta}_{M L E}=1 / \bar{Z}$ in Equation (2.3) for comparison of other estimates. Since the ML estimates of $\alpha$ and $\beta$ are the solution of the non-linear equation system for our model, to use the ML estimate of the unknown parameter of the random sample of the exponential distribution can be preferable with respect to the error performances. It is observed that Bayes estimate under the informative prior has the smallest error values in most cases. The performances of two ML estimates are very similar to each other. Hence, the MLE2 estimate can be used as an alternative estimates of $R_{C o m p}$. Similar to the Table 1, Bayes estimate under the non-informative prior has bigger error values than other estimates. Moreover, all the error values of the estimates are large when $R_{\text {Comp }}$ is around 0.5 , and it is small when $R_{C o m p}$ is close to extreme values 0 and 1 . This has also been obtained in similar stress-strength reliability studies in the literature, one can refer to $[7,25]$.

In Table 3, we have presented ML and nonparametric estimates of $R_{C o m p}$ and their relative efficiency for different parameters and sample sizes. The relative efficiency of $\widetilde{R}_{C o m p}$ relative to $\widehat{R}_{C o m p}$ is evaluated as ratio of corresponding MSEs, i.e. $R E=$ $\operatorname{MSE}\left(\widehat{R}_{\text {Comp }}\right) / M S E\left(\widetilde{R}_{\text {Comp }}\right)$. From Table $3, R E$ values are smaller than 1 in all cases, hence the ML estimate performance is better than the corresponding nonparametric estimate. Since the MSEs of these estimates are getting close to each other as the sample size increases, $\widetilde{R}_{\text {Comp }}$ could be used under the violation of distributional assumptions.

Table 3. ML and nonparametric estimates of $R_{C o m p}$ and relative efficiency.

\begin{tabular}{|c|c|c|c|c|c|c|c|c|c|}
\hline \multirow[b]{2}{*}{$n$} & \multirow[b]{2}{*}{$m$} & \multirow[b]{2}{*}{$R_{\text {Comp }}$} & \multicolumn{3}{|c|}{$M L E$} & \multicolumn{3}{|c|}{ Nonparametric } & \multirow[b]{2}{*}{$\mathrm{RE}$} \\
\hline & & & $\widehat{R}_{\text {Comp }}$ & Bias & MSE & $\widetilde{R}_{\text {Comp }}$ & Bias & MSE & \\
\hline \multicolumn{10}{|c|}{$(\alpha, \beta, \theta)=(1.5,4,6)$} \\
\hline \multirow[t]{5}{*}{5} & 10 & 0.73678 & 0.72797 & -0.00881 & 0.00847 & 0.72964 & -0.00714 & 0.01242 & 0.68226 \\
\hline & 20 & & 0.73199 & -0.00479 & 0.00426 & 0.73186 & -0.00492 & 0.00587 & 0.72684 \\
\hline & 40 & & 0.73617 & -0.00061 & 0.00213 & 0.73552 & -0.00126 & 0.00299 & 0.71097 \\
\hline & 80 & & 0.73649 & -0.00029 & 0.00113 & 0.73584 & -0.00094 & 0.00147 & 0.76795 \\
\hline & 100 & & 0.73692 & 0.00014 & 0.00091 & 0.73666 & -0.00012 & 0.00121 & 0.75136 \\
\hline \multirow[t]{5}{*}{10} & 10 & 0.62067 & 0.62112 & 0.00045 & 0.01001 & 0.61013 & -0.01054 & 0.01702 & 0.58814 \\
\hline & 20 & & 0.62233 & 0.00166 & 0.00520 & 0.61749 & -0.00318 & 0.00813 & 0.63911 \\
\hline & 40 & & 0.62268 & 0.00201 & 0.00254 & 0.61953 & -0.00113 & 0.00378 & 0.67376 \\
\hline & 80 & & 0.62194 & 0.00127 & 0.00133 & 0.62017 & -0.00049 & 0.00195 & 0.67965 \\
\hline & 100 & & 0.62132 & 0.00065 & 0.00108 & 0.61967 & -0.00099 & 0.00159 & 0.67732 \\
\hline \multicolumn{10}{|c|}{$(\alpha, \beta, \theta)=(5,3,15)$} \\
\hline \multirow[t]{4}{*}{3} & 10 & 0.89234 & 0.86835 & -0.02399 & 0.00438 & 0.87596 & -0.01638 & 0.00610 & 0.71802 \\
\hline & 25 & & 0.87886 & -0.01348 & 0.00158 & 0.88196 & -0.01038 & 0.00215 & 0.73148 \\
\hline & 50 & & 0.88463 & -0.00772 & 0.00071 & 0.88557 & -0.00677 & 0.00106 & 0.67620 \\
\hline & 100 & & 0.88847 & -0.00387 & 0.00033 & 0.88963 & -0.00271 & 0.00048 & 0.67162 \\
\hline \multirow[t]{4}{*}{6} & 10 & 0.81960 & 0.80174 & -0.01785 & 0.00600 & 0.80427 & -0.01532 & 0.00965 & 0.62247 \\
\hline & 25 & & 0.81208 & -0.00751 & 0.00233 & 0.81167 & -0.00792 & 0.00373 & 0.62518 \\
\hline & 50 & & 0.81266 & -0.00694 & 0.00121 & 0.81288 & -0.00672 & 0.00180 & 0.66983 \\
\hline & 100 & & 0.81649 & -0.00311 & 0.00059 & 0.81652 & -0.00308 & 0.00091 & 0.65199 \\
\hline \multicolumn{10}{|c|}{$(\alpha, \beta, \theta)=(1.1,1.25,0.5)$} \\
\hline \multirow[t]{4}{*}{4} & 10 & 0.27521 & 0.27647 & 0.00126 & 0.00591 & 0.26029 & -0.01492 & 0.01324 & 0.44654 \\
\hline & 20 & & 0.27028 & -0.00493 & 0.00275 & 0.26341 & -0.01181 & 0.00654 & 0.42125 \\
\hline & 40 & & 0.27115 & -0.00406 & 0.00139 & 0.26663 & -0.00858 & 0.00333 & 0.41865 \\
\hline & 60 & & 0.27120 & -0.00401 & 0.00097 & 0.26721 & -0.00800 & 0.00241 & 0.40168 \\
\hline \multirow[t]{4}{*}{8} & 10 & 0.19552 & 0.19666 & 0.00114 & 0.00341 & 0.18429 & -0.01124 & 0.01093 & 0.31203 \\
\hline & 20 & & 0.19425 & -0.00127 & 0.00169 & 0.18890 & -0.00662 & 0.00561 & 0.30082 \\
\hline & 40 & & 0.19219 & -0.00334 & 0.00077 & 0.18841 & -0.00712 & 0.00268 & 0.28603 \\
\hline & 60 & & 0.19303 & -0.00249 & 0.00052 & 0.18908 & -0.00644 & 0.00185 & 0.28094 \\
\hline
\end{tabular}


Table 4. Estimates of $\Phi_{C o m p}$ for $(\alpha, \beta, \theta)=(1.5,4,6)$.

\begin{tabular}{|c|c|c|c|c|c|c|c|c|c|c|c|}
\hline \multirow[b]{2}{*}{$n$} & \multirow[b]{2}{*}{$m$} & \multirow[b]{2}{*}{$\Phi_{\text {Comp }}$} & \multicolumn{3}{|c|}{$M L E$} & \multicolumn{3}{|c|}{ Bayes (Inf. prior) } & \multicolumn{3}{|c|}{ Bayes (Non-inf. prior) } \\
\hline & & & $\widehat{\Phi}_{\text {Comp }}$ & Bias & MSE & $\widehat{\Phi}_{\text {Comp }}^{\text {Lindley }}$ & Bias & ER & $\widehat{\Phi}_{\text {Comp }}^{\text {Lindley }}$ & Bias & ER \\
\hline \multirow[t]{5}{*}{8} & 20 & 0.17188 & 0.17054 & -0.00134 & 00024 & 0.17649 & 0.00461 & 00036 & 0.17530 & 0.00342 & 0.00062 \\
\hline & 40 & & 6 & -0.00031 & 012 & 17338 & 0.00150 & 014 & 0.17335 & 0.00147 & 0.00020 \\
\hline & 60 & & 19 & 0.00031 & 009 & 17311 & 0123 & 10 & 0.17318 & 0.00 & 0.00012 \\
\hline & 80 & & 10 & 0.00022 & 0007 & 17283 & 00095 & 11 & 0.17288 & 0.00 & 00013 \\
\hline & 100 & & 26 & 0.00038 & 00005 & 0.17264 & 0.00076 & 005 & 0.17268 & 0.00080 & 0.00006 \\
\hline \multirow[t]{5}{*}{12} & 20 & 0.1 & 0.13 & -0.00079 & 0.00010 & 0.13279 & 0.00158 & 0013 & 0.13248 & 0.00127 & 0.00021 \\
\hline & 40 & & $0.1:$ & 0.00030 & 0.00005 & 0.13303 & 0.00182 & 0.00100 & 0.13311 & 0.00189 & 0.00105 \\
\hline & 60 & & 0.13153 & 0.00032 & 0.00004 & 0.13186 & 0.00065 & 0.00004 & 0.13191 & 0.00070 & 0.00005 \\
\hline & 80 & & 0.13140 & 0.00018 & 0.00003 & 0.13159 & 0.00037 & 0.00003 & 0.13160 & 0.00039 & 0.00003 \\
\hline & 100 & & 0.13131 & 0.00009 & 0.00002 & 0.13143 & -0.00022 & 0002 & 0.13144 & 0.00023 & 0.00002 \\
\hline \multirow[t]{5}{*}{16} & 20 & 0.10 & 90 & -0.00011 & 0.00006 & 0.11069 & 0.00167 & 092 & 0.11076 & 0.00175 & 0.00097 \\
\hline & 40 & & 0.10922 & 0.00020 & 0003 & 0.10971 & 0.00070 & 0.00018 & 0.10976 & 0.00075 & 0.00020 \\
\hline & 60 & & 08 & 0.00008 & 0002 & 0.10922 & 0.00021 & 002 & 0.10923 & 0.00022 & 0.00002 \\
\hline & 80 & & 0.10910 & 0.00009 & 0.00001 & 0.10920 & 0.00018 & 001 & 0.10921 & 0.00019 & 0.00002 \\
\hline & 100 & & 0.10918 & 0.00016 & 0.00001 & 0.10924 & 0.00022 & 001 & 0.10925 & 23 & 0.00001 \\
\hline \multirow[t]{5}{*}{20} & 20 & 0.09472 & 70 & -0.00002 & 0004 & 0.09658 & 186 & 304 & 0.09669 & 97 & 0.00327 \\
\hline & 40 & & 85 & 0.00013 & 002 & 0.09516 & 44 & 06 & 0.09520 & 0. & 0.00007 \\
\hline & 60 & & 87 & 0.00015 & 001 & 0.09488 & 16 & & 0.09490 & 0. & 0.00002 \\
\hline & 80 & & 0.09491 & 0.00019 & 0.00001 & 0.09498 & 0.0 & 001 & 0.09499 & 0.0 & 0.00001 \\
\hline & 100 & & 0.09480 & 0.00007 & 0.00004 & 0.09483 & 0.00011 & 000 & 0.09484 & 0.0 & 0.00000 \\
\hline \multirow[t]{5}{*}{24} & 20 & 0.08 & 0.08 & -0.00008 & 0.00002 & 0.08580 & 0.00119 & 0076 & 0.08590 & 0.0 & 0.00082 \\
\hline & 40 & & 0.08477 & 0.00015 & 0.00001 & 0.08555 & 0.00094 & 0.00084 & 0.08559 & 0.00098 & 0.00087 \\
\hline & 60 & & 0.08476 & 0.00015 & 0.00001 & 0.08495 & 0.00033 & 0.00006 & 0.08497 & 0.00036 & 0.00007 \\
\hline & 80 & & 0.08471 & 0.00010 & 0.00001 & 0.08476 & 0.00014 & 0.00001 & 0.08476 & 0.00015 & 0.00001 \\
\hline & 100 & & 0.08466 & 0.00005 & 0.00000 & 0.08469 & 0.00008 & 0.00000 & 0.08470 & 0.00008 & 0.00000 \\
\hline
\end{tabular}

Table 5. Estimates of $\Phi_{C o m p}$ for $(\alpha, \beta, \theta)=(1.5,0.5,10)$.

\begin{tabular}{|c|c|c|c|c|c|c|c|c|c|c|c|}
\hline \multirow[b]{2}{*}{$n$} & \multirow[b]{2}{*}{$m$} & \multirow[b]{2}{*}{$\Phi_{\text {Comp }}$} & \multicolumn{3}{|c|}{$M L E$} & \multicolumn{3}{|c|}{ Bayes (Inf. prior) } & \multicolumn{3}{|c|}{ Bayes (Non-inf. prior) } \\
\hline & & & $\widehat{\Phi}_{\text {Comp }}$ & Bias & MSE & $\widehat{\Phi}_{\text {Comp }}^{\text {Lindley }}$ & Bias & ER & $\widehat{\Phi}_{\text {Comp }}^{\text {Lindley }}$ & Bias & ER \\
\hline \multirow[t]{5}{*}{5} & 10 & 0.77878 & 0.76043 & -0.01834 & .01219 & 0.81123 & .03246 & 0.01579 & 0.78802 & 0.00 & 0.02 \\
\hline & 20 & & 76574 & -0.01304 & 00634 & .79147 & 01269 & & 0.78422 & 0.00 & 0.01 \\
\hline & 30 & & 77424 & -0.00453 & 00485 & 0.78916 & 1039 & & 0.78675 & 0.00 & 0.00 \\
\hline & 40 & & 0.77578 & -0.00300 & 00356 & 0.78563 & & & 0.78474 & 0.00 & 0.00 \\
\hline & 50 & & 0.77783 & -0.00095 & 00305 & 0.78437 & & & 0.78398 & 0.0 & 0.0 \\
\hline \multirow[t]{5}{*}{10} & 10 & & 0 & -0.0 & 00353 & 0.49410 & 1 & & 0.48 & & \\
\hline & 20 & & 0.48 & -0.00190 & & 0.49303 & & & 0.49 & & \\
\hline & 30 & & 0.48 & 0.00 & & 0.49077 & & & 0.49079 & & \\
\hline & 40 & & & & & 0.48989 & & & 0.49 & & \\
\hline & 50 & & 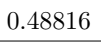 & & & 5 & & & 66 & & \\
\hline \multirow[t]{5}{*}{15} & 10 & & & & & & & & & & \\
\hline & 20 & & & -0.0 & & 0.37604 & & & 42 & & \\
\hline & 30 & & & & & 0.37 & & & & & \\
\hline & 40 & & & & & 0.37558 & & & 0.3 & & \\
\hline & 50 & & 0.37 & 0.0 & & 0.37 & & & 0.3 & & \\
\hline \multirow[t]{5}{*}{20} & 10 & & 6 & -0.0 & & 70 & & & 0.3 & & \\
\hline & 20 & & 0.31 & -0.00001 & & 0.31263 & & & 0.3 & & 0.00181 \\
\hline & 30 & & & 0.00012 & & & & & 0.31 & & \\
\hline & 40 & & & & & & & & 0.312 & & \\
\hline & 50 & & 0.31211 & 0.00025 & & 0.31185 & 0.00001 & & 0.31 & & 0.00023 \\
\hline \multirow[t]{5}{*}{25} & 10 & 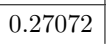 & 0.26943 & -0.00129 & 0.00062 & 0.27669 & 0.00596 & & 0.27925 & 0.00852 & 0.08576 \\
\hline & 20 & & 0.27080 & 0.00008 & & 0.27411 & 0.00339 & & 0.27470 & 0.00397 & 0.00791 \\
\hline & 30 & & 0.27174 & 0.00101 & & 0.27162 & 0.00090 & & 0.27203 & 0.00130 & 0.00062 \\
\hline & 40 & & 0.27144 & 0.00071 & & 0.27099 & 0.00027 & & 0.27121 & 0.00049 & 0.00019 \\
\hline & 50 & & 0.27129 & 0.00056 & 0.00014 & 0.27095 & 0.00023 & 0.00013 & 0.27108 & 0.00036 & 0.00015 \\
\hline
\end{tabular}


In Tables 4 and 5, we have listed the ML and Bayesian Lindley estimates of $\Phi_{C o m p}$ based on informative and non-informative priors. The true values of the parameters are taken as $(\alpha, \beta, \theta)=(1.5,4,6)$ and $(1.5,0.5,10)$ for $n=8,12,16,20,24$ and $5,10,15,20,25$, respectively. From Tables 4 and 5, we conclude that ML estimates of $\Phi_{C o m p}$ have relatively better results than Bayes estimates. However, ERs of Bayes estimate based on informative prior are close to MSEs of ML estimate as the sample size increases. From Table 6, we observe that AL of the asymptotic confidence intervals of $\Phi_{\text {Comp }}$ decreases as the sample size increases as expected, and all the CPs are satisfactory.

Table 6. Average lengths and coverage probabilities of $\Phi_{\text {Comp }}$ for $(\alpha, \beta, \theta)=(1.5,4,6)$ and $(1.5,0.5,10)$.

\begin{tabular}{l|l|l|ll|l|l|l|ll}
\hline$n$ & $m$ & $\Phi_{\text {Comp }}$ & $\mathrm{AL}$ & $\mathrm{CP}$ & $n$ & $m$ & $\Phi_{\text {Comp }}$ & $\mathrm{AL}$ & $\mathrm{CP}$ \\
\hline 8 & 20 & 0.17188 & 0.06319 & 0.9504 & 5 & 10 & 0.77878 & 0.49134 & 0.9564 \\
& 40 & & 0.04459 & 0.9544 & & 20 & & 0.34783 & 0.9600 \\
& 60 & & 0.03649 & 0.9548 & & 30 & & 0.28497 & 0.9504 \\
& 80 & & 0.03160 & 0.9472 & & 40 & & 0.24706 & 0.9596 \\
& 100 & & 0.02826 & 0.9472 & & 50 & & 0.22136 & 0.9544 \\
\hline 12 & 20 & 0.13121 & 0.04187 & 0.9592 & 10 & 10 & 0.48849 & 0.25506 & 0.9592 \\
& 40 & & 0.02960 & 0.9528 & & 20 & & 0.18026 & 0.9460 \\
& 60 & & 0.02416 & 0.9472 & & 30 & & 0.14749 & 0.9552 \\
& 80 & & 0.02091 & 0.9436 & & 40 & & 0.12775 & 0.9496 \\
& 100 & & 0.01871 & 0.9456 & & 50 & & 0.11424 & 0.9504 \\
\hline 16 & 20 & 0.10901 & 0.03125 & 0.9648 & 15 & 10 & 0.37502 & 0.17181 & 0.9528 \\
& 40 & & 0.02207 & 0.9540 & & 20 & & 0.12134 & 0.9572 \\
& 60 & & 0.01797 & 0.9524 & & 30 & & 0.09924 & 0.9540 \\
& 80 & & 0.01555 & 0.9508 & & 40 & & 0.08603 & 0.9480 \\
& 100 & & 0.01391 & 0.9516 & & 50 & & 0.07706 & 0.9468 \\
\hline 20 & 20 & 0.09472 & 0.02515 & 0.9616 & 20 & 10 & 0.31185 & 0.12999 & 0.9580 \\
& 40 & & 0.01752 & 0.9612 & & 20 & & 0.09311 & 0.9552 \\
& 60 & & 0.01425 & 0.9524 & & 30 & & 0.07509 & 0.9492 \\
& 80 & & 0.01234 & 0.9440 & & 40 & & 0.06496 & 0.9468 \\
& 100 & & 0.01103 & 0.9484 & & 50 & & 0.05808 & 0.9452 \\
\hline 24 & 20 & 0.08461 & 0.02097 & 0.9644 & 25 & 10 & 0.27072 & 0.10507 & 0.9556 \\
& 40 & & 0.01462 & 0.9520 & & 20 & & 0.07542 & 0.9524 \\
& 60 & & 0.01190 & 0.9512 & & 30 & & 0.06064 & 0.9588 \\
& 80 & & 0.01022 & 0.9472 & & 40 & & 0.05224 & 0.9512 \\
& 100 & & 0.00913 & 0.9492 & & 50 & & 0.04671 & 0.9456 \\
\hline
\end{tabular}

In Tables 7, 9 and 10, we have presented the ML, nonparametric and Bayesian MCMC estimates of $R_{\text {System }}$ and $\Phi_{\text {System }}$ based on informative and non-informative priors. In Tables 8 and 11, we have reported an \%95 asymptotic confidence interval and HPD credible intervals based on informative and non-informative priors. The true values of the parameters are taken as $(\alpha, \beta, \theta)=(1.5,2.5,15),(6,2,3)$ and $(3,1,10)$ for $n=2,4,8$, and $3,5,7$ and $3,8,12,16$, respectively. According to Table $7, \mathrm{MCMC}$ estimate of $R_{\text {System }}$ based on informative prior gives better result than non-informative prior and MLE in terms of ER and MSEs. We also note that MSE, ER, and bias of the estimates tend to decrease generally as the sample size increases. Table 8 indicates that AL of HPD credible intervals based on informative prior are mostly narrower than the others. CPs of asymptotic confidence interval and HPD credible interval based on informative prior are quite satisfactory. However, in some cases, it is observed that CPs of HPD credible interval based on non-informative prior are far away from the nominal value 0.95. All the intervals tend to become narrower as the sample size increases. 
Table 7. Estimates of $R_{\text {System }}$.

\begin{tabular}{|c|c|c|c|c|c|c|c|c|c|c|c|}
\hline & & & \multicolumn{3}{|c|}{$M L E$} & \multicolumn{3}{|c|}{ Bayes (Inf. prior) } & \multicolumn{3}{|c|}{ Bayes (Non-inf. prior) } \\
\hline$n$ & $m$ & $R_{\text {System }}$ & $\widehat{R}_{\text {System }}$ & Bias & MSE & $\widehat{R}_{\text {System }}^{M C M C}$ & Bias & ER & $\widehat{R}_{\text {System }}^{M C M C}$ & Bias & ER \\
\hline \multicolumn{12}{|c|}{$(\alpha, \beta, \theta)=(1.5,2.5,15)$} \\
\hline \multirow[t]{4}{*}{2} & 10 & 0.95833 & 0.93500 & -0.02333 & 0.00199 & 0.93220 & -0.02613 & 0.00108 & 0.89034 & -0.06800 & 0.00640 \\
\hline & 20 & & 0.94298 & -0.01536 & 0.00093 & 0.93892 & -0.01941 & 0.00062 & 0.89957 & -0.05876 & 0.00422 \\
\hline & 30 & & 0.94618 & -0.01215 & 0.00061 & 0.94176 & -0.01657 & 0.00046 & 0.90108 & -0.05725 & 0.00382 \\
\hline & 40 & & 0.94889 & -0.00944 & 0.00042 & 0.94445 & -0.01388 & 0.00035 & 0.90361 & -0.05473 & 0.00340 \\
\hline \multirow[t]{4}{*}{4} & 10 & 0.88571 & 0.85353 & -0.03218 & 0.00551 & 0.84447 & -0.04124 & 0.00317 & 0.79729 & -0.08843 & 0.01214 \\
\hline & 20 & & 0.86370 & -0.02202 & 0.00273 & 0.85547 & -0.03025 & 0.00185 & 0.80856 & -0.07715 & 0.00799 \\
\hline & 30 & & 0.86769 & -0.01802 & 0.00183 & 0.85976 & -0.02596 & 0.00139 & 0.81108 & -0.07463 & 0.00691 \\
\hline & 40 & & 0.87031 & -0.01540 & 0.00140 & 0.86287 & -0.02285 & 0.00113 & 0.81262 & -0.07309 & 0.00637 \\
\hline 8 & 10 & 0.74 & 0.71911 & -0.02692 & 0.00931 & 0.70264 & -0.04339 & 0.00472 & 0.66527 & -0.08077 & 0.01356 \\
\hline \multirow{2}{*}{8} & 20 & & 0.72145 & -0.02459 & 0.00549 & 0.71189 & -0.03415 & 0.00335 & 0.67112 & -0.07492 & 0.00942 \\
\hline & 30 & & 0.72785 & -0.01817 & 0.00374 & 0.71809 & -0.02794 & 0.00257 & 0.67523 & -0.07080 & 0.00764 \\
\hline & 40 & & 0.73065 & -0.01538 & 0.00264 & 0.72167 & -0.02436 & 0.00200 & 0.67667 & -0.06936 & 0.00669 \\
\hline \multicolumn{12}{|c|}{$(\alpha, \beta, \theta)=(6,2,3)$} \\
\hline \multirow[t]{4}{*}{3} & 10 & 0.42857 & 0.43662 & 0.00805 & 0.01096 & 0.41822 & -0.01035 & 620 & 0.41333 & -0.0 & 0.00914 \\
\hline & 20 & & 0.42963 & 0.00106 & 0.00564 & 0.42101 & -0.00756 & 0.00404 & 0.41156 & -0.01701 & 0.00491 \\
\hline & 30 & & 0.42767 & -0.00090 & 0.00380 & 0.42076 & -0.00782 & 0.00296 & 0.40928 & -0.01929 & 0.00348 \\
\hline & 40 & & 0.42880 & 0.00023 & 0.00294 & 0.42270 & -0.00587 & 0.00239 & 0.41028 & -0.01829 & 0.00276 \\
\hline \multirow[t]{4}{*}{5} & 10 & 0.30070 & 0.31192 & 0.01122 & 0.00822 & 0.29893 & -0.00177 & 0.00442 & 0.30076 & 0.00006 & 0.00685 \\
\hline & 20 & & 0.30523 & 0.00453 & 0.00407 & 0.30005 & -0.00065 & 0.00287 & 0.29691 & -0.00379 & 0.00348 \\
\hline & 30 & & 0.30475 & 0.00405 & 0.00276 & 0.30090 & 0.00020 & 0.00215 & 0.29600 & -0.00470 & 0.00237 \\
\hline & 40 & & 0.30286 & 0.00216 & 0.00212 & 0.29951 & -0.00119 & 0.00174 & 0.29364 & -0.00706 & 0.00187 \\
\hline \multirow[t]{4}{*}{7} & 10 & 0.23137 & 0.24538 & 0.01401 & 0.00623 & 0.23500 & 0.00362 & 0.00333 & 0.23959 & 0.00821 & 0.00527 \\
\hline & 20 & & 0.23687 & 0.00550 & 0.00288 & 0.23333 & 0.00195 & 0.00204 & 0.23275 & 0.00138 & 0.00251 \\
\hline & 30 & & 0.23585 & 0.00448 & 0.00197 & 0.23354 & 0.00216 & 0.00155 & 0.23136 & -0.00001 & 0.00174 \\
\hline & 40 & & 0.23389 & 0.00252 & 0.00145 & 0.23205 & 0.00068 & 0.00120 & 0.22900 & -0.00237 & 0.00129 \\
\hline \multicolumn{12}{|c|}{$(\alpha, \beta, \theta)=(3,1,10)$} \\
\hline \multirow[t]{4}{*}{3} & 10 & 0.89069 & 86467 & -0.02602 & 0.00466 & 0.85425 & -0.03644 & 0.00292 & 0.81347 & -0.07722 & 0.00986 \\
\hline & 20 & & 0.87467 & -0.01601 & 0.00238 & 0.86338 & -0.02730 & 0.00175 & 0.82398 & -0.06671 & 0.00635 \\
\hline & 30 & & 0.87745 & -0.01324 & 0.00161 & 0.86625 & -0.02443 & 0.00134 & 0.82496 & -0.06573 & 0.00553 \\
\hline & 40 & & 0.88015 & -0.01054 & 0.00120 & 0.86943 & -0.02126 & 0.00108 & 0.82728 & -0.06341 & 0.00497 \\
\hline \multirow[t]{4}{*}{8} & 10 & 0.68627 & 0.66996 & -0.01631 & 0.01055 & 0.65079 & -0.03548 & 0.00556 & 0.62519 & -0.06108 & 0.01225 \\
\hline & 20 & & 0.67488 & -0.01140 & 0.00573 & 0.66104 & -0.02523 & 0.00360 & 0.63176 & -0.05452 & 0.00741 \\
\hline & 30 & & 0.67730 & -0.00898 & 0.00373 & 0.66482 & -0.02145 & 0.00267 & 0.63321 & -0.05307 & 0.00571 \\
\hline & 40 & & 0.67731 & -0.00897 & 0.00300 & 0.66572 & -0.02056 & 0.00232 & 0.63210 & -0.05417 & 0.00516 \\
\hline \multirow[t]{4}{*}{12} & 10 & 0.57312 & 0.56849 & -0.00463 & 0.01154 & 0.54860 & -0.02452 & 0.00540 & 0.53273 & -0.04039 & 0.01073 \\
\hline & 20 & & 0.56501 & -0.00811 & 0.00631 & 0.55263 & -0.02049 & 0.00378 & 0.53165 & -0.04147 & 0.00659 \\
\hline & 30 & & 0.56960 & -0.00352 & 0.00414 & 0.55765 & -0.01547 & 0.00278 & 0.53468 & -0.03844 & 0.00469 \\
\hline & 40 & & 0.56853 & -0.00460 & 0.00342 & 0.55865 & -0.01447 & 0.00247 & 0.53365 & -0.03947 & 0.00424 \\
\hline \multirow[t]{4}{*}{16} & 10 & 0.49071 & 0.49218 & 0.00147 & 0.01154 & 0.47313 & -0.01758 & 0.00504 & 0.46464 & -0.02607 & 0.00985 \\
\hline & 20 & & 0.48786 & -0.00285 & 0.00604 & 0.47677 & -0.01395 & 0.00348 & 0.46241 & -0.02831 & 0.00563 \\
\hline & 30 & & 0.48829 & -0.00243 & 0.00425 & 0.47908 & -0.01164 & 0.00275 & 0.46204 & -0.02867 & 0.00417 \\
\hline & 40 & & 0.48929 & -0.00143 & 0.00326 & 0.48076 & -0.00995 & 0.00230 & 0.46202 & -0.02869 & 0.00340 \\
\hline
\end{tabular}


Table 8. Average lengths and coverage probabilities of $R_{\text {System }}$.

\begin{tabular}{|c|c|c|c|c|c|c|c|c|}
\hline \multirow[b]{2}{*}{$n$} & \multirow[b]{2}{*}{$m$} & \multirow[b]{2}{*}{$R_{\text {System }}$} & \multicolumn{2}{|c|}{ Asymptotic } & \multicolumn{2}{|c|}{ HPD (Inf. prior) } & \multicolumn{2}{|c|}{ HPD (Non-inf. prior) } \\
\hline & & & $\mathrm{AL}$ & $\mathrm{CP}$ & $\mathrm{AL}$ & $\mathrm{CP}$ & $\mathrm{AL}$ & $\mathrm{CP}$ \\
\hline \multicolumn{9}{|c|}{$(\alpha, \beta, \theta)=(1.5,2.5,15)$} \\
\hline \multirow[t]{4}{*}{2} & 10 & 0.95833 & 0.17487 & 0.9640 & 0.11561 & 0.9972 & 0.17728 & 0.7904 \\
\hline & 20 & & 0.11381 & 0.9628 & 0.09018 & 0.9916 & 0.12449 & 0.5484 \\
\hline & 30 & & 0.08917 & 0.9748 & 0.07851 & 0.9884 & 0.10427 & 0.3632 \\
\hline & 40 & & 0.07423 & 0.9688 & 0.06998 & 0.9900 & 0.09210 & 0.2816 \\
\hline \multirow[t]{4}{*}{4} & 10 & 0.88571 & 0.29261 & 0.9588 & 0.21676 & 0.9932 & 0.28100 & 0.8876 \\
\hline & 20 & & 0.20376 & 0.9562 & 0.17112 & 0.9488 & 0.19995 & 0.7176 \\
\hline & 30 & & 0.16490 & 0.9616 & 0.14935 & 0.9876 & 0.16671 & 0.6116 \\
\hline & 40 & & 0.14170 & 0.9592 & 0.13464 & 0.9868 & 0.14730 & 0.4980 \\
\hline \multirow[t]{4}{*}{8} & 10 & 0.74603 & 0.40249 & 0.9484 & 0.31619 & 0.9904 & 0.37564 & 0.9308 \\
\hline & 20 & & 0.29071 & 0.9444 & 0.25256 & 0.9824 & 0.27272 & 0.8700 \\
\hline & 30 & & 0.23800 & 0.9412 & 0.22001 & 0.9784 & 0.22543 & 0.8272 \\
\hline & 40 & & 0.20681 & 0.9580 & 0.19859 & 0.9828 & 0.19800 & 0.7692 \\
\hline \multicolumn{9}{|c|}{$(\alpha, \beta, \theta)=(6,2,3)$} \\
\hline \multirow[t]{4}{*}{3} & 10 & 0.42857 & 0.42022 & 0.9324 & 0.37913 & 0.9712 & 0.41144 & 0.9552 \\
\hline & 20 & & 0.30100 & 0.9440 & 0.28668 & 0.9700 & 0.29596 & 0.9568 \\
\hline & 30 & & 0.24683 & 0.9448 & 0.24024 & 0.9664 & 0.24235 & 0.9548 \\
\hline & 40 & & 0.21460 & 0.9500 & 0.21188 & 0.9656 & 0.21070 & 0.9472 \\
\hline \multirow[t]{4}{*}{5} & 10 & 0.30070 & 0.35666 & 0.9340 & 0.32110 & 0.9752 & 0.35610 & 0.9572 \\
\hline & 20 & & 0.25214 & 0.9416 & 0.24157 & 0.9688 & 0.25390 & 0.9612 \\
\hline & 30 & & 0.20629 & 0.9488 & 0.20201 & 0.9700 & 0.20728 & 0.9592 \\
\hline & 40 & & 0.17805 & 0.9484 & 0.17691 & 0.9652 & 0.17890 & 0.9568 \\
\hline \multirow[t]{4}{*}{7} & 10 & 0.23137 & 0.30498 & 0.9296 & 0.27387 & 0.9720 & 0.30904 & 0.9604 \\
\hline & 20 & & 0.21225 & 0.9508 & 0.20387 & 0.9748 & 0.21697 & 0.9660 \\
\hline & 30 & & 0.17301 & 0.9428 & 0.17004 & 0.9660 & 0.17641 & 0.9592 \\
\hline & 40 & & 0.14889 & 0.9516 & 0.14846 & 0.9656 & 0.15193 & 0.9616 \\
\hline \multicolumn{9}{|c|}{$(\alpha, \beta, \theta)=(3,1,10)$} \\
\hline \multirow[t]{4}{*}{3} & 10 & 0.89069 & 0.27973 & 0.9556 & 0.21038 & 0.9908 & 0.26180 & 0.9096 \\
\hline & 20 & & 0.19395 & 0.9556 & 0.16615 & 0.9852 & 0.18698 & 0.8052 \\
\hline & 30 & & 0.15795 & 0.9576 & 0.14597 & 0.9868 & 0.15650 & 0.6664 \\
\hline & 40 & & 0.13610 & 0.9576 & 0.13174 & 0.9868 & 0.13792 & 0.5804 \\
\hline \multirow[t]{4}{*}{8} & 10 & 0.68627 & 0.42078 & 0.9380 & 0.34216 & 0.9868 & 0.38808 & 0.9364 \\
\hline & 20 & & 0.30398 & 0.9456 & 0.27039 & 0.9792 & 0.28263 & 0.9228 \\
\hline & 30 & & 0.25102 & 0.9528 & 0.23481 & 0.9800 & 0.23486 & 0.9032 \\
\hline & 40 & & 0.21867 & 0.9484 & 0.21163 & 0.9760 & 0.20630 & 0.8648 \\
\hline \multirow[t]{4}{*}{12} & 10 & 0.57312 & 0.44064 & 0.9464 & 0.36177 & 0.9880 & 0.40890 & 0.9496 \\
\hline & 20 & & 0.31877 & 0.9476 & 0.28567 & 0.9788 & 0.29917 & 0.9284 \\
\hline & 30 & & 0.26295 & 0.9512 & 0.24700 & 0.9784 & 0.24801 & 0.9336 \\
\hline & 40 & & 0.22848 & 0.9400 & 0.22159 & 0.9708 & 0.21677 & 0.9048 \\
\hline \multirow[t]{4}{*}{16} & 10 & 0.49072 & 0.43492 & 0.9312 & 0.35790 & 0.9844 & 0.40695 & 0.9440 \\
\hline & 20 & & 0.31331 & 0.9428 & 0.28182 & 0.9776 & 0.29723 & 0.9468 \\
\hline & 30 & & 0.25773 & 0.9396 & 0.24273 & 0.9768 & 0.24588 & 0.9360 \\
\hline & 40 & & 0.22418 & 0.9424 & 0.21761 & 0.9736 & 0.21450 & 0.9284 \\
\hline
\end{tabular}


Table 9. ML and nonparametric estimates of $R_{\text {System }}$ and relative efficiency.

\begin{tabular}{|c|c|c|c|c|c|c|c|c|c|}
\hline \multirow[b]{2}{*}{$n$} & \multirow[b]{2}{*}{$m$} & \multirow[b]{2}{*}{$R_{\text {System }}$} & \multicolumn{3}{|c|}{$M L E$} & \multicolumn{3}{|c|}{ Nonparametric } & \multirow[b]{2}{*}{$\mathrm{RE}$} \\
\hline & & & $\widehat{R}_{\text {System }}$ & Bias & MSE & $\widetilde{R}_{\text {System }}$ & Bias & MSE & \\
\hline \multicolumn{10}{|c|}{$(\alpha, \beta, \theta)=(1.5,2.5,15)$} \\
\hline \multirow[t]{5}{*}{3} & 10 & 0.92308 & 0.89514 & -0.02794 & 0.00350 & 0.89848 & -0.02460 & 0.00471 & 0.74310 \\
\hline & 20 & & 0.90225 & -0.02083 & 0.00190 & 0.90787 & -0.01521 & 0.00204 & 0.93217 \\
\hline & 40 & & 0.90930 & -0.01377 & 0.00094 & 0.91385 & -0.00923 & 0.00096 & 0.97985 \\
\hline & 80 & & 0.91550 & -0.00758 & 0.00039 & 0.91848 & -0.00460 & 0.00041 & 0.95857 \\
\hline & 100 & & 0.91726 & -0.00582 & 0.00032 & 0.91915 & -0.00393 & 0.00035 & 0.91624 \\
\hline \multirow[t]{5}{*}{6} & 10 & 0.81250 & 0.78207 & -0.03043 & 0.00776 & 0.77801 & -0.03449 & 0.01024 & 0.75781 \\
\hline & 20 & & 0.78829 & -0.02421 & 0.00427 & 0.79158 & -0.02092 & 0.00481 & 0.88739 \\
\hline & 40 & & 0.79485 & -0.01765 & 0.00231 & 0.79796 & -0.01454 & 0.00239 & 0.96530 \\
\hline & 80 & & 0.80250 & -0.01000 & 0.00109 & 0.80439 & -0.00810 & 0.00114 & 0.95630 \\
\hline & 100 & & 0.80394 & -0.00856 & 0.00087 & 0.80559 & -0.00691 & 0.00090 & 0.96693 \\
\hline \multicolumn{10}{|c|}{$(\alpha, \beta, \theta)=(1.25,0.75,2)$} \\
\hline \multirow[t]{4}{*}{5} & 10 & 0.50593 & 0.50155 & -0.00437 & 0.01160 & 0.48538 & -0.02055 & 0.01579 & 0.73423 \\
\hline & 25 & & 0.50081 & -0.00512 & 0.00500 & 0.49544 & -0.01049 & 0.00668 & 0.74891 \\
\hline & 50 & & 0.50059 & -0.00534 & 0.00258 & 0.49835 & -0.00757 & 0.00329 & 0.78543 \\
\hline & 100 & & 0.50297 & -0.00296 & 0.00125 & 0.50193 & -0.00400 & 0.00160 & 0.78236 \\
\hline \multirow[t]{4}{*}{10} & 10 & 0.31942 & 0.32743 & 0.00801 & 0.00835 & 0.31459 & -0.00483 & 0.01369 & 0.60973 \\
\hline & 25 & & 0.32065 & 0.00123 & 0.00340 & 0.31651 & -0.00291 & 0.00553 & 0.61470 \\
\hline & 50 & & 0.31902 & -0.00040 & 0.00171 & 0.31654 & -0.00288 & 0.00301 & 0.56752 \\
\hline & 100 & & 0.31937 & -0.00005 & 0.00088 & 0.31831 & -0.00111 & 0.00145 & 0.60584 \\
\hline \multicolumn{10}{|c|}{$(\alpha, \beta, \theta)=(5,4,20)$} \\
\hline \multirow[t]{4}{*}{7} & 10 & 0.62879 & 0.60628 & -0.02251 & 0.01165 & 0.59318 & -0.03561 & 0.01575 & 0.73946 \\
\hline & 20 & & 0.60885 & -0.01994 & 0.00615 & 0.60567 & -0.02311 & 0.00763 & 0.80560 \\
\hline & 40 & & 0.61695 & -0.01184 & 0.00315 & 0.61654 & -0.01225 & 0.00366 & 0.86228 \\
\hline & 60 & & 0.61840 & -0.01039 & 0.00222 & 0.61773 & -0.01106 & 0.00254 & 0.87294 \\
\hline \multirow[t]{4}{*}{14} & 10 & 0.42690 & 0.42672 & -0.00018 & 0.01002 & 0.41210 & -0.01480 & 0.01466 & 0.68345 \\
\hline & 20 & & 0.41992 & -0.00698 & 0.00527 & 0.41135 & -0.01555 & 0.00789 & 0.66786 \\
\hline & 40 & & 0.42296 & -0.00395 & 0.00284 & 0.42029 & -0.00661 & 0.00411 & 0.69081 \\
\hline & 60 & & 0.42322 & -0.00368 & 0.00182 & 0.42109 & -0.00581 & 0.00263 & 0.69271 \\
\hline
\end{tabular}

ML and nonparametric estimates of $R_{\text {System }}$, and their relative efficiency $R E=$ $\operatorname{MSE}\left(\widehat{R}_{\text {System }}\right) / M S E\left(\widetilde{R}_{\text {System }}\right)$ are given in Table 9 . It is seen that the performance of these estimates is similar to the component level case. $R E$ values are very close to 1 in some cases. Hence, larger sample sizes were also considered in these cases, but the $R E$ values did not exceed 1 .

From Table 10, we see that performances of the MLE and MCMC estimate of $\Phi_{\text {System }}$ based on informative prior are similar. In some cases, MSEs of ML estimates are smaller than ERs of MCMC estimates, and vice versa. Hence, we can not give a general order for these estimates. However, these errors are close to each other as the sample size increases. From Table 11, we observe that AL of the asymptotic confidence intervals is generally smaller than that of both HPD credible intervals. Furthermore, AL of all the intervals decreases as the sample size increases, and all CPs are quite satisfactory.

Moreover, some plots are presented for easily comparing the performance of the estimates of $R_{\text {System }}$. In Figure 3, ML and MCMC Bayesian (under the informative prior) estimates of $R_{\text {System }}$ are plotted for different parameters and sizes. It is observed that Bayes estimate under the informative prior has smaller error than that of ML estimate similar to the Table 7 . 
Table 10. Estimates of $\Phi_{\text {System }}$.

\begin{tabular}{|c|c|c|c|c|c|c|c|c|c|c|c|}
\hline & & & \multicolumn{3}{|c|}{$M L E$} & \multicolumn{3}{|c|}{ Bayes (Inf. prior) } & \multicolumn{3}{|c|}{ Bayes (Non-inf. prior) } \\
\hline$n$ & $m$ & $\Phi_{\text {System }}$ & $\widehat{\Phi}_{\text {System }}$ & Bias & MSE & $\widehat{\Phi}_{\text {System }}^{M C M C}$ & Bias & ER & $\widehat{\Phi}_{\text {System }}^{M C M C}$ & Bias & ER \\
\hline \multicolumn{12}{|c|}{$(\alpha, \beta, \theta)=(1.5,2.5,15)$} \\
\hline \multirow[t]{4}{*}{2} & 10 & 0.48986 & 0.50810 & 0.01825 & 0.01510 & 0.51325 & 0.02340 & 0.01255 & 0.56352 & 0.07366 & 0.02344 \\
\hline & 20 & & 0.50222 & 0.01236 & 0.00745 & 0.50699 & 0.01714 & 0.00678 & 0.54361 & 0.05376 & 0.01108 \\
\hline & 30 & & 0.49983 & 0.00997 & 0.00498 & 0.50423 & 0.01438 & 0.00469 & 0.53750 & 0.04765 & 0.00756 \\
\hline & 40 & & 0.49850 & 0.00864 & 0.00367 & 0.50211 & 0.01226 & 0.00351 & 0.53372 & 0.04386 & 0.00583 \\
\hline \multirow[t]{4}{*}{4} & 10 & 41 & 0.24933 & 0.01493 & 0.00386 & 0.25146 & 0.01706 & 0.00327 & 0.28236 & 0.04795 & 0.00687 \\
\hline & 20 & & 0.24478 & 0.01037 & 0.00184 & 0.24732 & 0.01291 & 0.00170 & 0.27173 & 0.03732 & 0.00338 \\
\hline & 30 & & 0.24185 & 0.00744 & 0.00117 & 0.24437 & 0.00996 & 0.00114 & 0.26723 & 0.03282 & 0.00234 \\
\hline & 40 & & 0.24103 & 0.00662 & 0.00091 & 0.24337 & 0.00896 & 0.00089 & 0.26571 & 0.03130 & 0.00195 \\
\hline \multirow[t]{4}{*}{8} & 10 & 0.11206 & 0.12193 & 0.00988 & 0.00090 & 0.12288 & 0.01082 & 0.00078 & 0.14087 & 0.02881 & 0.00187 \\
\hline & 20 & & 0.11940 & 0.00734 & 0.00050 & 0.12048 & 0.00843 & 0.00046 & 0.13544 & 0.02338 & 0.00106 \\
\hline & 30 & & 0.11754 & 0.00548 & 0.00031 & 0.11890 & 0.00685 & 0.00030 & 0.13329 & 0.02124 & 0.00078 \\
\hline & 40 & & 7 & 0.00451 & 0.00024 & 0.11795 & 0.00589 & 0.00023 & 0.13222 & & 0.00065 \\
\hline \multicolumn{12}{|c|}{$(\alpha, \beta, \theta)=(6,2,3)$} \\
\hline \multirow[t]{4}{*}{3} & 10 & 0.18518 & 20419 & 0.01900 & 0.00322 & 0.20540 & 0.02022 & 0.00260 & 0.23341 & 0.04823 & 0.00589 \\
\hline & 20 & & 0.19845 & 0.01326 & 0.00156 & 0.20227 & 0.01708 & 0.00147 & 0.22498 & 0.03980 & 0.00314 \\
\hline & 30 & & 0.19397 & 0.00878 & 0.00105 & 0.19935 & 0.01416 & 0.00105 & 0.22055 & 0.03536 & 0.00231 \\
\hline & 40 & & & 0.00702 & & 0.19780 & & 0.00080 & 0.21842 & & \\
\hline \multirow[t]{4}{*}{5} & 10 & 0.11008 & 0.12169 & 0.01161 & 0.00115 & 0.12261 & 0.01254 & 0.00095 & 0.13997 & 0.02989 & 0.00219 \\
\hline & 20 & & 0.11759 & 0.00751 & 0.00057 & 0.12001 & 0.00993 & 0.00053 & 0.13415 & 0.02407 & 0.00114 \\
\hline & 30 & & 0.11577 & 0.00569 & 0.00040 & 0.11903 & 0.00895 & 0.00041 & 0.13233 & 0.02225 & 0.00091 \\
\hline & 40 & & 0.11435 & 0.00427 & 0.00029 & 0.11803 & 0.00796 & 0.00031 & 0.13116 & 0.02108 & 0.00074 \\
\hline \multirow[t]{4}{*}{7} & 10 & 0.07829 & & 0.00855 & 061 & 3742 & & & 0.10006 & & 0.00 \\
\hline & 20 & & 0.08355 & 0.00526 & 0.00029 & 0.08545 & 0.00716 & 0.00028 & 0.09569 & 0.01740 & 0.00061 \\
\hline & 30 & & 0.08255 & 0.00426 & 0.00020 & 0.08483 & 0.00655 & 0.00020 & 0.09447 & 0.01618 & 0.00046 \\
\hline & 40 & & 0.08152 & 0.00323 & 0.00015 & 0.08417 & 0.00588 & 0.00016 & 0.09358 & & 0.00038 \\
\hline \multicolumn{12}{|c|}{$(\alpha, \beta, \theta)=(3,1,10)$} \\
\hline \multirow[t]{4}{*}{3} & 10 & 0.39899 & 0.42901 & 0.03002 & 0.01292 & 0.44142 & 0.04243 & 0.01321 & 0.48432 & 0.08533 & 0.02240 \\
\hline & 20 & & 0.41915 & 0.02016 & 0.00650 & 0.42812 & 0.02913 & 0.00676 & 0.46161 & 0.06262 & 0.01081 \\
\hline & 30 & & 0.40992 & 0.01093 & 0.00402 & 0.41761 & 0.01862 & 0.00415 & 0.44913 & 0.05014 & 0.00682 \\
\hline & 40 & & 0.40809 & 0.00910 & 0.00311 & 0.41486 & 0.01587 & 0.00323 & 0.44566 & 0.04667 & 0.00546 \\
\hline \multirow[t]{4}{*}{8} & 10 & 0.14286 & 0.15399 & 0.01113 & 0.00170 & 0.15880 & 0.01595 & 0.00174 & 0.17769 & 0.03483 & 0.00320 \\
\hline & 20 & & 0.15168 & 0.00882 & 0.00088 & 0.15547 & 0.01262 & 0.00094 & 0.17165 & 0.02879 & 0.00177 \\
\hline & 30 & & 0.15009 & 0.00723 & 0.00064 & 0.15370 & 0.01084 & 0.00068 & 0.16927 & 0.02642 & 0.00134 \\
\hline & 40 & & 0.14696 & 0.00410 & 0.00044 & 0.15034 & 0.00748 & 0.00046 & 0.16587 & 0.02302 & 0.00100 \\
\hline \multirow[t]{4}{*}{12} & 10 & 0.09387 & 0.10265 & 0.00878 & 0.00079 & 0.10594 & 0.01207 & 0.00082 & 0.11950 & 0.02563 & 0.00158 \\
\hline & 20 & & 0.09952 & 0.00565 & 0.00039 & 0.10224 & 0.00837 & 0.00042 & 0.11366 & 0.01979 & 0.00081 \\
\hline & 30 & & 0.09800 & 0.00413 & 0.00026 & 0.10077 & 0.00690 & 0.00028 & 0.11194 & 0.01807 & 0.00060 \\
\hline & 40 & & 0.09733 & 0.00346 & 0.00020 & 0.09975 & 0.00588 & 0.00021 & 0.11096 & 0.01709 & 0.00049 \\
\hline \multirow[t]{4}{*}{16} & 10 & 0.06982 & 0.07641 & 0.00659 & 0.00044 & 0.07896 & 0.00914 & 0.00046 & 0.08946 & 0.01964 & 0.00091 \\
\hline & 20 & & 0.07417 & 0.00435 & 0.00022 & 0.07622 & 0.00640 & 0.00023 & 0.08507 & 0.01525 & 0.00046 \\
\hline & 30 & & 0.07345 & 0.00363 & 0.00015 & 0.07548 & 0.00566 & 0.00016 & 0.08418 & 0.01436 & 0.00036 \\
\hline & 40 & & 0.07261 & 0.00279 & 0.00117 & 0.07461 & 0.00479 & 0.00013 & 0.08339 & 0.01357 & 0.00303 \\
\hline
\end{tabular}


Table 11. Average lengths and coverage probabilities of $\Phi_{\text {System }}$

\begin{tabular}{|c|c|c|c|c|c|c|c|c|}
\hline \multirow[b]{2}{*}{$n$} & \multirow[b]{2}{*}{$m$} & \multirow[b]{2}{*}{$\Phi_{\text {System }}$} & \multicolumn{2}{|c|}{ Asymptotic } & \multicolumn{2}{|c|}{ HPD (Inf. prior) } & \multicolumn{2}{|c|}{ HPD (Non-inf. prior) } \\
\hline & & & $\mathrm{AL}$ & $\mathrm{CP}$ & $\mathrm{AL}$ & $\mathrm{CP}$ & $\mathrm{AL}$ & $\mathrm{CP}$ \\
\hline \multicolumn{9}{|c|}{$(\alpha, \beta, \theta)=(1.5,2.5,15)$} \\
\hline \multirow[t]{4}{*}{2} & 10 & 0.48986 & 0.56874 & 0.9620 & 0.58449 & 0.9936 & 0.71627 & 0.9964 \\
\hline & 20 & & 0.38614 & 0.9652 & 0.42406 & 0.9900 & 0.47991 & 0.9928 \\
\hline & 30 & & 0.30793 & 0.9656 & 0.35356 & 0.9916 & 0.38510 & 0.9908 \\
\hline & 40 & & 0.26294 & 0.9672 & 0.31009 & 0.9936 & 0.33028 & 0.9904 \\
\hline \multirow[t]{4}{*}{4} & 10 & 0.23441 & 0.28581 & 0.9676 & 0.28971 & 0.9916 & 0.36000 & 0.9932 \\
\hline & 20 & & 0.19345 & 0.9687 & 0.20992 & 0.9512 & 0.24057 & 0.9512 \\
\hline & 30 & & 0.15352 & 0.9772 & 0.17427 & 0.9940 & 0.19242 & 0.9872 \\
\hline & 40 & & 0.13109 & 0.9724 & 0.15308 & 0.9928 & 0.16569 & 0.9744 \\
\hline \multirow[t]{4}{*}{8} & 10 & 0.11206 & 0.14426 & 0.9796 & 0.14248 & 0.9952 & 0.18005 & 0.9924 \\
\hline & 20 & & 0.09849 & 0.9772 & 0.10352 & 0.9912 & 0.12056 & 0.9836 \\
\hline & 30 & & 0.07771 & 0.9804 & 0.08597 & 0.9924 & 0.09651 & 0.9692 \\
\hline & 40 & & 0.06604 & 0.9788 & 0.07536 & 0.9944 & 0.08310 & 0.9488 \\
\hline \multicolumn{9}{|c|}{$(\alpha, \beta, \theta)=(6,2,3)$} \\
\hline \multirow[t]{4}{*}{3} & 10 & 0.18518 & 0.25661 & 0.9760 & 0.25099 & 0.9928 & 0.31665 & 0.9912 \\
\hline & 20 & & 0.17554 & 0.9788 & 0.17901 & 0.9916 & 0.20879 & 0.9824 \\
\hline & 30 & & 0.13921 & 0.9728 & 0.14606 & 0.9884 & 0.16538 & 0.9592 \\
\hline & 40 & & 0.11918 & 0.9732 & 0.12727 & 0.9856 & 0.14127 & 0.9508 \\
\hline \multirow[t]{4}{*}{5} & 10 & 0.11008 & 0.15535 & 0.9772 & 0.15012 & 0.9916 & 0.19003 & 0.9908 \\
\hline & 20 & & 0.10559 & 0.9776 & 0.10674 & 0.9936 & 0.12488 & 0.9792 \\
\hline & 30 & & 0.08468 & 0.9792 & 0.08785 & 0.9860 & 0.09949 & 0.9472 \\
\hline & 40 & & 0.07218 & 0.9716 & 0.07652 & 0.9876 & 0.08500 & 0.9272 \\
\hline \multirow[t]{4}{*}{7} & 10 & 0.07829 & 0.11151 & 0.9796 & 0.10716 & 0.9952 & 0.13589 & 0.9900 \\
\hline & 20 & & 0.07567 & 0.9796 & 0.07616 & 0.9916 & 0.08907 & 0.9732 \\
\hline & 30 & & 0.06091 & 0.9764 & 0.06276 & 0.9864 & 0.07103 & 0.9512 \\
\hline & 40 & & 0.05205 & 0.9720 & 0.05483 & 0.9800 & 0.06084 & 0.9312 \\
\hline \multicolumn{9}{|c|}{$(\alpha, \beta, \theta)=(3,1,10)$} \\
\hline \multirow[t]{4}{*}{3} & 10 & 0.39899 & 0.50010 & 0.9628 & 0.52019 & 0.9884 & 0.60748 & 0.9908 \\
\hline & 20 & & 0.33781 & 0.9640 & 0.36919 & 0.9844 & 0.40582 & 0.9820 \\
\hline & 30 & & 0.26611 & 0.9640 & 0.29954 & 0.9892 & 0.32152 & 0.9796 \\
\hline & 40 & & 0.22839 & 0.9596 & 0.26066 & 0.9836 & 0.27609 & 0.9664 \\
\hline \multirow[t]{4}{*}{8} & 10 & 0.14286 & 0.18717 & 0.9700 & 0.18994 & 0.9908 & 0.22384 & 0.9920 \\
\hline & 20 & & 0.12862 & 0.9800 & 0.13698 & 0.9880 & 0.15153 & 0.9748 \\
\hline & 30 & & 0.10322 & 0.9656 & 0.11319 & 0.9848 & 0.12205 & 0.9532 \\
\hline & 40 & & 0.08682 & 0.9632 & 0.09736 & 0.9844 & 0.10367 & 0.9464 \\
\hline \multirow[t]{4}{*}{12} & 10 & 0.09387 & 0.12669 & 0.9736 & 0.12742 & 0.9900 & 0.15040 & 0.9880 \\
\hline & 20 & & 0.08601 & 0.9744 & 0.09063 & 0.9840 & 0.10060 & 0.9748 \\
\hline & 30 & & 0.06858 & 0.9692 & 0.07488 & 0.9872 & 0.08088 & 0.9552 \\
\hline & 40 & & 0.05885 & 0.9676 & 0.06541 & 0.9876 & 0.06960 & 0.9316 \\
\hline \multirow[t]{4}{*}{16} & 10 & 0.06982 & 0.09541 & 0.9824 & 0.09514 & 0.9940 & 0.11296 & 0.9884 \\
\hline & 20 & & 0.06508 & 0.9780 & 0.06787 & 0.9872 & 0.07533 & 0.9732 \\
\hline & 30 & & 0.05228 & 0.9728 & 0.05638 & 0.9872 & 0.06092 & 0.9444 \\
\hline & 40 & & 0.04450 & 0.9696 & 0.04915 & 0.9836 & 0.05236 & 0.9172 \\
\hline
\end{tabular}



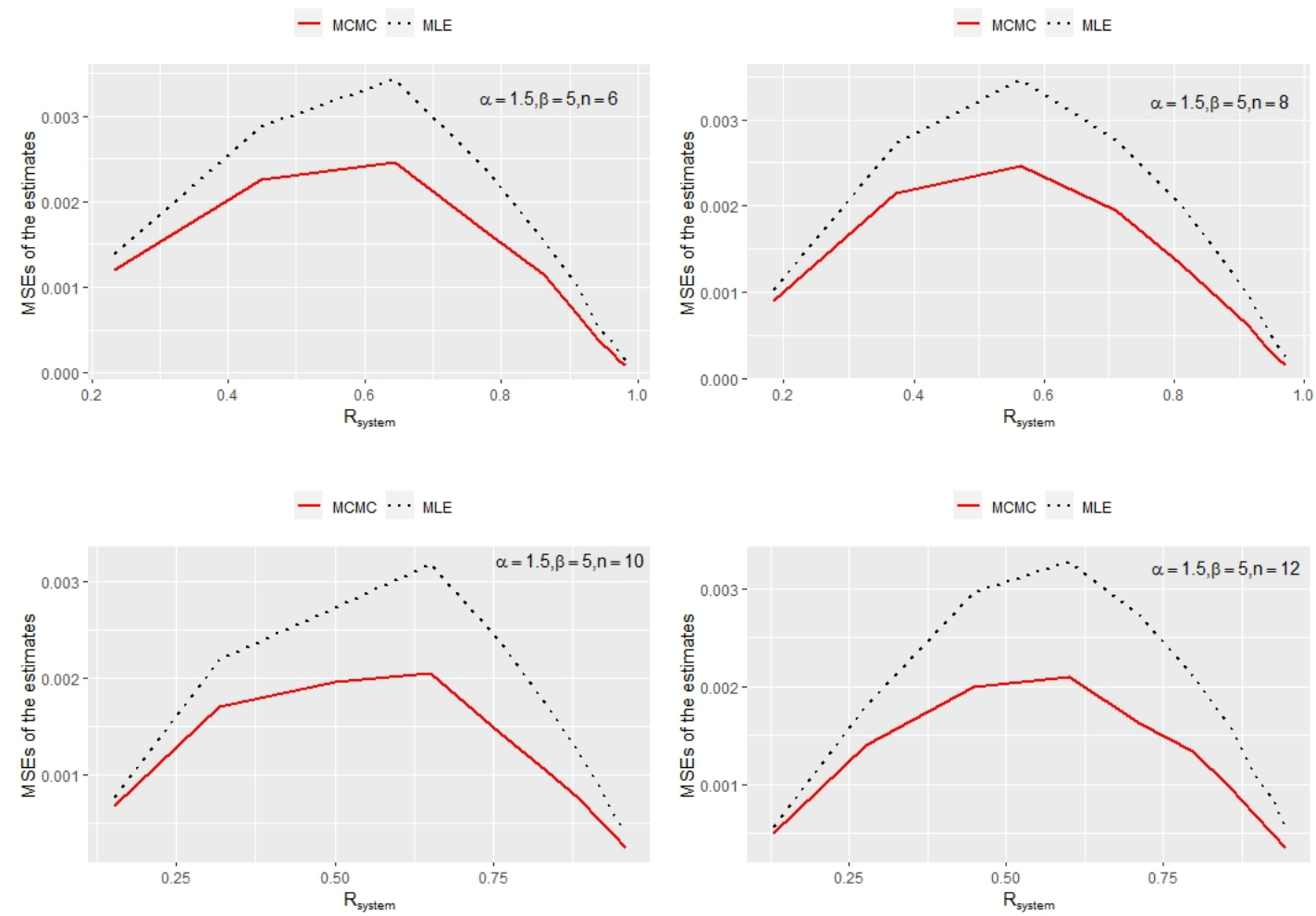

Figure 3. Plots for the $R_{\text {System }}$ estimates when $m=40$.

\section{Real data analysis}

In this section, the lifetime of steel specimens under the different stress levels has been considered for a real-life example. This data set represents the lifetime data for the steel specimens under fourteen different stress levels. All data sets are available in [28]. In the literature, these data sets have been studied many times by researchers for the stressstrength model.

We consider the series system which has $n$ active components with corresponding $n$ standby components. It is assumed that active $(\mathbf{X})$ and standby $(\mathbf{Y})$ components are tested at 32.5 and 35 stress levels. Let us assume that an engineer wants to compare that the series system which is constructed by $\mathbf{X}$ and $\mathbf{Y}$ with another component $(\mathbf{T})$ which is tested at 33 stress level. The reliability of this series system is estimated by using these data sets. Then, he/she decides that which system or component is used in the production processes. Different situations can be created according to this scenario. For example, if the reliability of this series system exceeds 0.80 , the series system with standby components will be preferred.

We have 20 observations for each data set. For two different $(n, m)$ cases, the original data is divided by 100 based on the aforementioned scenario. The strength data sets $\mathbf{X}$ and $\mathbf{Y}$ are partitioned into $m$ parts and each one has $n$ unit. $\mathbf{T}$ is obtaining as the average value of each part. We check whether data sets $\mathbf{X}, \mathbf{Y}, \mathbf{T}_{1}$ (stress data set for $n=4, m=5$ ) and $\mathbf{T}_{2}$ (stress data set for $n=5, m=4$ ) come from the exponential distribution or not. Kolmogorov-Smirnov (K-S), Anderson-Darling (A-D) and Cramer-von Mises (C-VM) tests are carried out for the goodness-of-fit test. Their test statistics values and corresponding $p$-values are listed in Table 12. It is observed that the exponential distribution provides a good fit to these data sets. 
Table 12. Goodnes-of-fit test for the real data set.

\begin{tabular}{cccccccc}
\hline Data & $M L E$ & $K-S$ & $p-$ value & $A-D$ & $p-$ value & $C-V M$ & $p$-value \\
\hline $\mathbf{X}$ & $\widehat{\alpha}=0.0907$ & 0.1629 & 0.6067 & 0.4343 & 0.8123 & 0.0503 & 0.8799 \\
\hline $\mathbf{Y}$ & $\widehat{\beta}=0.2909$ & 0.2843 & 0.0635 & 1.8888 & 0.1064 & 0.3439 & 0.1015 \\
\hline $\mathbf{T}_{1}$ & $\widehat{\theta}=0.1099$ & 0.5023 & 0.1090 & 1.4018 & 0.2017 & 0.2963 & 0.1372 \\
\hline $\mathbf{T}_{2}$ & $\widehat{\theta}=0.1099$ & 0.4726 & 0.2396 & 0.8944 & 0.4099 & 0.1816 & 0.3141 \\
\hline
\end{tabular}

The observed data at component level $\left(\mathbf{Z}, \mathbf{T}_{1}\right)$ for $n=4, m=5$ and $\mathbf{T}_{2}$ for $n=5$, $m=4$ are given by

$$
\mathbf{Z}=\left[\begin{array}{cccc}
44.87 & 10.48 & 9.77 & 16.59 \\
4.00 & 8.76 & 21.88 & 5.07 \\
6.52 & 9.95 & 22.55 & 6.78 \\
9.84 & 3.73 & 10.41 & 6.93 \\
20.47 & 26.42 & 11.18 & 33.06
\end{array}\right], \mathbf{T}_{1}=\left[\begin{array}{c}
6.3500 \\
7.9750 \\
8.9400 \\
13.3800 \\
8.8575
\end{array}\right] \mathbf{T}_{2}=\left[\begin{array}{c}
5.822 \\
7.094 \\
13.474 \\
10.012
\end{array}\right]
$$

In this case, the MLE of the parameters are $(\widehat{\alpha}, \widehat{\beta}, \widehat{\theta})=(0.1872,0.1096,0.1099)$. The ML and Bayes estimates of $R_{C o m p}$ and $\Phi_{C o m p}$ along with $95 \%$ asymptotic confidence and HPD credible intervals (given in bracket under the estimates) are presented in Table 13. We need to determine the hyperparameters for the Bayes estimate. If a practitioner has not any knowledge about the hyperparameters of the prior distributions, he/she can be use the moment estimates of the gamma distribution for each sample. Next, we present the results based on three different priors. The moment estimates of data sets $\mathbf{X}, \mathbf{Y}, \mathbf{T}_{1}$ are used as Prior 1: $a_{1}=1.1229, b_{1}=0.1018, a_{2}=2.1986, b_{2}=0.6395, a_{3}=15.2068(9.5378)$, $b_{3}=1.6710(1.0480)$ for $n=4(5), m=5(4)$. Then, Bayes estimates are computed based on the informative priors Prior 1, Prior 2: $a_{i}=b_{i}=1, i=1,2,3$ and non-informative prior Prior 3: $a_{i}=b_{i}=0, i=1,2,3$.

Table 13. Estimates of $R_{C o m p}$ and $\Phi_{C o m p}$ for the real data set.

\begin{tabular}{ccccccc}
\hline & $(n, m)$ & MLE & MLE2 & MCMC (Prior 1) & MCMC (Prior 2) & MCMC (Prior 3) \\
\hline$R_{\text {Comp }}$ & $(4,5)$ & 0.42711 & 0.40395 & 0.83871 & 0.60608 & 0.59900 \\
& & $(0.09230,0.76192)$ & $(0.08959,0.71831)$ & $(0.71668,0.95150)$ & $(0.35220,0.84807)$ & $(0.32209,0.86436)$ \\
$\Phi_{\text {Comp }}$ & 4.22900 & 4.00617 & 13.9646 & 13.36341 & 17.31083 \\
& - & $(3.87442,4.13791)$ & $(5.82671,29.75366)$ & $(5.71158,24.46485)$ & $(6.43095,37.48032)$ \\
\hline$R_{\text {Comp }}$ & $(5,4)$ & 0.38948 & 0.36612 & 0.80318 & 0.61540 & 0.60619 \\
& $(0.07360,0.70536)$ & $(0.07597,0.65627)$ & $(0.64387,0.94009)$ & $(0.34135,0.88631)$ & $(0.30222,0.90935)$ \\
$\Phi_{\text {Comp }}$ & 3.62949 & 3.40479 & 13.75202 & 14.07141 & 20.60584 \\
& - & $(3.35311,3.45647)$ & $(4.97322,29.62783)$ & $(5.33867,29.95478)$ & $(6.64036,51.85836)$ \\
\hline
\end{tabular}

The observed data at system level $\mathbf{Z}_{1}$ for $n=4, m=5$ and $\mathbf{Z}_{2}$ for $n=5, m=4$ are given by

$$
\mathbf{Z}_{1}=\min (\mathbf{X})+\min (\mathbf{Y})=\left[\begin{array}{c}
9.68 \\
3.42 \\
6.32 \\
3.73 \\
11.18
\end{array}\right], \mathbf{Z}_{2}=\left[\begin{array}{c}
4.00 \\
3.42 \\
3.73 \\
6.18
\end{array}\right]
$$


The stress data sets are the same as in component level case. In this case, the MLE of the parameters are $(\widehat{\alpha}, \widehat{\beta}, \widehat{\theta})=(0.07282,0.07281,0.10988)$ and $(0.092326,0.092325,0.109880)$ for $n=4, m=5$ and $n=5, m=4$, respectively, based on system level. The ML and Bayes estimates of $R_{\text {System }}$ and $\Phi_{\text {System }}$ along with $95 \%$ asymptotic confidence intervals and HPD credible intervals (given in bracket under the estimates) are listed in Table 14. Moreover, Bayes estimates are computed based on the same priors as in the component level.

Table 14. Estimates of $R_{\text {System }}$ and $\Phi_{\text {System }}$ for the real data set.

\begin{tabular}{ccccccc}
\hline & $(n, m)$ & MLE & MLE2 & MCMC (Prior 1) & MCMC (Prior 2) & MCMC (Prior 3) \\
\hline$R_{\text {System }}$ & $(4,5)$ & 0.47279 & 0.29870 & 0.70654 & 0.43288 & 0.42911 \\
& & $(0.16274,0.78283)$ & $(0.04816,0.54923)$ & $(0.52938,0.87513)$ & $(0.16807,0.72397)$ & $(0.16960,0.70405)$ \\
$\Phi_{\text {System }}$ & 5.42190 & 3.00456 & 6.45067 & 6.48932 & 8.10311 \\
& & $(1.91364,8.93015)$ & $(0.30430,5.70483)$ & $(2.48701,11.69452)$ & $(0.57635,12.20563)$ & $(2.83643,15.15230)$ \\
\hline$R_{\text {System }}$ & $(5,4)$ & 0.34757 & 0.25158 & 0.55486 & 0.33495 & 0.33862 \\
& & $(0.04645,0.64869)$ & $(0.00312,0.50004)$ & $(0.32318,0.78846)$ & $(0.08286,0.606934)$ & $(0.07420,0.63406)$ \\
$\Phi_{\text {System }}$ & 3.36457 & 2.39699 & 3.94688 & 4.12537 & 5.66156 \\
& $(0.95960,5.76955)$ & $(0,4.82559)$ & $(1.12749,7.56060)$ & $(0.66982,8.79625)$ & $(1.37796,11.58791)$ \\
\hline
\end{tabular}

\section{Conclusions}

In this study, statistical inference for the stress-strength reliability and MRS are considered for the series system when cold standby components are used both component level and system level. The classical and Bayesian approaches have been used to estimate the stress-strength reliability and MRS of the system. In Bayesian case, estimates are obtained by using Lindley's approximation and MCMC method.

Our simulation results show that Bayes estimate of the stress-strength reliability based on informative prior has better performance than other estimates. The ML estimate of the MRS generally provides better results as compared with Bayes estimates for small sample sizes. However, the performance of Bayes estimate based on informative prior gets closer to ML as the sample size increases. From the real data analysis, we observe that classical and Bayesian (based on informative prior) methods for both the stress-strength reliability and MRS have similar point and interval estimates.

In our model, the total lifetime of the strength component and corresponding standby component is a convolution of the two independent and non-identical random variables. It is known that the convolution of random variables have mixed form except for some wellknown distributions under the certain conditions. For this reason, when we consider the lifetime distributions except for the exponential one, we will encounter the lifetime of the related system that has not a closed form. Moreover, when the standby components are considered as warm standby in the system, the similar problem will arise. We will consider these problems as future studies. We hope to report our new results in this regard in the future.

\section{References}

[1] K. Ahmadi and S. Ghafouri, Reliability estimation in a multicomponent stressstrength model under generalized half-normal distribution based on progressive type-II censoring, J. Stat. Comput. Simul. 89 (13), 2505-2548, 2019.

[2] F.G. Akgül, Reliability estimation in multicomponent stress-strength model for ToppLeone distribution, J. Stat. Comput. Simul. 89 (15), 2914-2929, 2019. 
[3] E.K. AL-Hussaini, M.A.M.A. Mousa and K.S. Sultan, Parametric and nonparametric estimation of $P(Y<X)$ for finite mixtures of lognormal components, Comm. Statist. Theory Methods 26 (5), 1269-1289, 1997.

[4] M.M. Ali, M. Pal and J. Woo, Estimation of $P(Y<X)$ in a four-parameter generalized gamma distribution, Austrian J. Stat. 41 (3), 197-210, 2012.

[5] A. Baklizi and O. Eidous, Nonparametric estimation of $P(X<Y)$ using kernel methods, Metron 64 (1), 47-60, 2006.

[6] R.E. Barlow and F. Proschan, Statistical Theory of Reliability and Life Testing, International Series in Decision Processes, Holt, Rinehart and Winston Inc, 1975.

[7] M. Basirat, S. Baratpour and J. Ahmadi, Statistical inferences for stress-strength in the proportional hazard models based on progressive Type-II censored samples, J. Stat. Comput. Simul. 85 (3), 431-449, 2015.

[8] Z.W.Birnbaum, On a use of Mann-Whitney statistics, in: Proceedings of the 3rd Berkley Symposium in Mathematics, Statistics and Probability, 3 (1), 13-17, 1956.

[9] Z.W. Birnbaum and B.C. McCarty, A distribution-free upper confidence bounds for $\operatorname{Pr}(Y<X)$ based on independent samples of $X$ and $Y$, Ann. Math. Statist. 29 (2), 558-562, 1958.

[10] P.J. Boland and E. El-Neweihi E, Component redundancy vs system redundancy in the hazard rate ordering, IEEE Trans. Rel. 44 (4), 614-619, 1995.

[11] C. Cetinkaya and A.I. Genc, Stress-strength reliability estimation under the standard two-sided power distribution, Appl. Math. Model. 65, 72-88, 2019.

[12] J.H. Cha, J. Mi and W.Y. Yun, Modelling a general standby system and evaluation of its performance, Appl. Stoch. Models Bus. Ind. 24 (2), 159-169, 2008.

[13] J. Chen, Y. Zhang, P. Zhao and S. Zhou, Allocation strategies of standby redundancies in series/parallel system, Comm. Statist. Theory Methods 47 (3), 708-724, 2018.

[14] M.H. Chen and Q.M. Shao, Monte Carlo estimation of Bayesian credible and HPD intervals, J. Comput. Graph. Statist. 8 (1), 69-92, 1999.

[15] S. Eryilmaz, Dynamic reliability and performance evaluation of multi-state systems with two components, Hacet. J. Math. Stat. 62 (1), 125-133, 2011.

[16] S. Eryilmaz, Reliability of a k-out-of-n system equipped with a single warm standby component, IEEE Trans. Rel. 62 (2), 499-503, 2013.

[17] S. Eryilmaz, The effectiveness of adding cold standby redundancy to a coherent system at system and component levels, Reliab. Eng. Syst. Saf. 165, 331-335, 2017.

[18] A. Gelman, J.B. Carlin, H.S. Stern and D.B. Rubin, Bayesian Data Analysis, Chapman Hall, 2003.

[19] S. Gurler, The mean remaining strength of systems in a stress-strength model, Hacet. J. Math. Stat. 42 (2), 181-187, 2013.

[20] S. Gurler, B.H. Ucer and I. Bairamov, On the mean remaining strength at the system level for some bivariate survival models based on exponential distribution, J. Comput. Appl. Math. 290, 535-542, 2015.

[21] B. Hasselman, Package "nleqslv", R package version: 3.3.2, 2018.

[22] N.K. Hazra and A.K. Nanda, Component redundancy versus system redundancy in different stochastic orderings, IEEE Trans. Rel. 63 (2), 567-582, 2014.

[23] M. Jovanovıć, B. Milošević and M. Obradović, Estimation of stress-strength probability in a multicomponent model based on geometric distribution,Hacet. J. Math. Stat. 49 (4), 1515-1532, 2020.

[24] T. Kayal, Y.M. Tripathi, S. Dey and S. Wu, On estimating the reliability in a multicomponent stress-strength model based on Chen distribution, Comm. Statist. Theory Methods 49 (10), 2429-2447, 2019.

[25] F. Kizilaslan, Classical and Bayesian estimation of reliability in a multicomponent stress-strength model based on a general class of inverse exponentiated distributions, Statist. Papers 59 (3), 1161-1192, 2018. 
[26] F. Kizilaslan, The mean remaining strength of parallel systems in a stress-strength model based on exponential distribution, Commun. Fac. Sci. Univ. Ank. Ser. A1 Math. Stat. 68 (2), 1435-1451, 2019.

[27] C. S. Kumar, Standby redundancy at system and component levels-A comparison, Microelectron. Reliab 35 (4), 751-752, 1995.

[28] J. Lawless, Statistical Models and Methods for Lifetime Data, 2nd ed., Wiley, New York, 2003.

[29] D.V. Lindley, Approximate Bayes method, Trabajos de Estadistica 3, 281-288, 1980.

[30] Y. Liu, Y. Shi, X. Bai and P. Zhan, Reliability estimation of a $N$-M-cold-standby redundancy system in a multicomponent stress-strength model with generalized halflogistic distribution, Physica A 490, 231-249, 2018.

[31] M. Mahdizadeh, On estimating a stress-strength type reliability, Hacet. J. Math. Stat. 47 (1), 243-253, 2018.

[32] M. Mahdizadeh and E. Zamanzade, Kernel-based estimation of $P(X>Y)$ in ranked set sampling, SORT 40 (2), 243-266, 2016.

[33] R. Nojosa and P.N. Rathie, Stress-strength reliability models involving generalized gamma and Weibull distributions, Int. J. Qual. Reliab. 37 (4), 538-551, 2020.

[34] R Core Team R, A language and environment for statistical computing, Vienna, Austria, R Foundation for Statistical Computing, 2020.

[35] C.R. Rao, Linear Statistical Inference and Its Applications, Wiley, 1965.

[36] T.R. Rasethuntsa and M. Nadar, Stress-strength reliability of a non-identicalcomponent-strengths system based on upper record values from the family of Kumaraswamy generalized distributions, Statistics 52 (3), 684-716, 2018.

[37] K. Shen and M. Xie, The effectiveness of adding standby redundancy at system and component levels, IEEE Trans. Rel. 40 (1), 53-55, 1991.

[38] K.C. Siju and M. Kumar, Estimation of stress-strength reliability of a parallel system with active, warm and cold standby components, J. Ind. Prod. Eng. 34 (8), 590-610, 2017.

[39] L. Tierney, Markov chains for exploring posterior distributions, Ann. Statist. 22 (4), 1701-1728, 1994.

[40] R. Yan, B. Lu and X. Li, On Redundancy allocation to series and parallel systems of two components, Comm. Statist. Theory Methods 48 (18), 4690-4701, 2019.

[41] P. Zhao, Y. Zhang and L. Li, Redundancy allocation at component level versus system level, European J. Oper. Res. 241 (2), 402-411, 2015. 